\title{
Scaling solutions and weak gravity in dark energy with energy and momentum couplings
}

\author{
Luca Amendola ${ }^{1}$ and Shinji Tsujikawa ${ }^{2}$ \\ ${ }^{1}$ Institute of Theoretical Physics, Heidelberg University, \\ Philosophenweg 16, 69120 Heidelberg, Germany and \\ ${ }^{2}$ Department of Physics, Faculty of Science, Tokyo University of Science, \\ 1-3, Kagurazaka, Shinjuku-ku, Tokyo 162-8601, Japan
}

(Dated: June 4, 2020)

\begin{abstract}
We argue that the $\Lambda$ CDM tensions of the Hubble-Lemaitre expansion rate $H_{0}$ and the clustering normalization $\sigma_{8}$ can be eased, at least in principle, by considering an interaction between dark energy and dark matter in such a way to induce a small and positive early effective equation of state and a weaker gravity. For a dark energy scalar field $\phi$ interacting with dark matter through an exchange of both energy and momentum, we derive a general form of the Lagrangian allowing for the presence of scaling solutions. In a subclass of such interacting theories, we show the existence of a scaling $\phi$-matter-dominated-era $(\phi \mathrm{MDE})$ which can potentially alleviate the $H_{0}$ tension by generating an effective high-redshift equation of state. We also study the evolution of perturbations for a model with $\phi \mathrm{MDE}$ followed by cosmic acceleration and find that the effective gravitational coupling relevant to the linear growth of large-scale structures can be smaller than the Newton gravitational constant $G$ at low redshifts. The momentum exchange between dark energy and dark matter plays a crucial role for realizing weak gravity, while the energy transfer is also required for the existence of $\phi \mathrm{MDE}$.
\end{abstract}

\section{INTRODUCTION}

Testing gravitational interactions with cosmological and astrophysical observations is one of the most interesting area of current research. Several large-scale surveys that will contribute to this task are underway or planned for the next few years [1 [ ] . If there is a deviation from Einstein gravity, then new observable phenomena are expected to appear, including stronger or weaker gravitational clustering and lensing, scale-dependent perturbation growth, screening of fifth forces, breaking of the equivalence principle, anomalous propagation of gravitational waves, and so on.

In order to predict these new effects, one often analyses the most general gravity theories with second-order equations of motion-like scalar-tensor [7-12, vector-tensor [13-16, massive gravity [17, 18] or, alternatively, proceeds by building effective Lagrangians that include all possible viable operators 19 23. These approaches have the great virtue of being systematic, but they rapidly lead to a proliferation of operators that make difficult to extract definite predictions. The gravitational-wave event GW170817 24], together with its electromagnetic counterpart [25, put additional constraints on the allowed Lagrangian [26 31, but there is still degeneracy among different dark energy models. This is partly attributed to the fact that, for the theories in which the speed of gravity is equivalent to that of light, the effective gravitational coupling for cold-dark-matter (CDM) density perturbations is usually larger than the Newton gravitational constant $G$ in both scalar-tensor and vector-tensor theories $32 \sqrt{34}$.

In this paper, we proceed in a different way as compared to the theoretical approach mentioned above. We begin by identifying which are the physical effects we are interested in, and then build a general model that is expected to generate them. The physical effects we consider are motivated by the two most interesting discrepancies between the

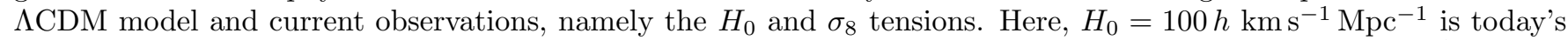
Hubble-Lemaitre expansion rate and $\sigma_{8}$ is the amplitude of matter perturbations within the comoving $8 h^{-1}$ scale.

As is well-known, the $H_{0}$ tension arises because the estimate of $H_{0}$ from Cosmic Microwave Background (CMB) temperature anisotropies [35] differs by more than $3 \sigma$ from the one based on its local measurements [6]. While there remains the possibility that this tension is due to unknown or uncontrolled systematic effects, the problem has been confirmed and exacerbated in recent observations [36-39]. Similarly, the $\sigma_{8}$ tension, which is weaker than the $H_{0}$ tension, is due to the difference between the clustering normalization obtained from CMB and the one from shear lensing analyses [40, 41, where the former favours values of $\sigma_{8}$ larger than the latter. In both cases, the CMB estimate depends on assuming $\Lambda \mathrm{CDM}$ as a model of dark energy and dark matter. It is therefore natural to ask whether a suitable modification of the dark sector can lead to a better agreement with the data.

One way to reconcile, at least potentially, $H_{0} \mathrm{CMB}$ results [35] with a higher local value [6], is to modify the evolution of the Universe between matter-radiation equality and cosmic acceleration, so that the effective early equation of state is slightly positive. In this case, one can show that the CMB acoustic peaks move to smaller angular scales, and therefore a higher $H_{0}$ is needed to bring the model back to agreement with observations (although of course a full likelihood analysis is needed to assess the valid parameter space). One way to achieve this without altering the 
physical properties of matter is to couple dark matter to dark energy in such a way that a matter-dominated epoch with a small fraction of dark energy is present. Indeed, for a canonical scalar-field dark energy model interacting with dark matter through the energy transfer characterized by a coupling constant $Q$ [42, 43, there exists a scaling $\phi$-matter-dominated-era ( $\phi \mathrm{MDE})$ during which the field density parameter $\Omega_{\phi}$ is constant $\left(\Omega_{\phi}=2 Q^{2} / 3\right)$ 43. During the $\phi \mathrm{MDE}$ the scalar-field kinetic energy dominates over its potential energy, yielding a field equation of state $w_{\phi}=1$. In this case, a small and positive effective equation of state arises, such that $w_{\text {eff }}=w_{\phi} \Omega_{\phi}=\Omega_{\phi}$. A comparison with cosmological datasets has put upper limits to $Q$ [44, 45].

In the models of Refs. [42, 43, the dark energy scalar field $\phi$ interacts with CDM (but not baryons) via a conformal rescaling of the metric. This type of couplings arises in Brans-Dicke theories [46] after a conformal transformation to the Einstein frame [47-49]. In the language of Schutz-Sorkin action [50, 51] describing CDM as a perfect fluid, the interacting Lagrangian for the models of Refs. 42, 43] is of the form $L_{\mathrm{int}}=\left(e^{Q \phi / M_{\mathrm{pl}}}-1\right) \rho_{m}$ [52], where $\rho_{m}$ is the CDM density and $M_{\mathrm{pl}}$ is the reduced Planck mass. Reflecting the fact that this interaction corresponds to the energy transfer, the effective gravitational coupling $G_{\text {eff }}$ associated with the linear evolution of CDM perturbations is larger than $G$, such that $G_{\text {eff }}=\left(1+2 Q^{2}\right) G[53$.

One problem for the models with $G_{\text {eff }}>G$ is that the attractive gravitational force induced by the coupling increases the cosmic growth rate, which makes the $\sigma_{8}$ tension worse. Ideally, one would need a coupling such that the $\phi \mathrm{MDE}$ is accompanied or followed by weaker growth of matter perturbations. An effective gravitational coupling smaller than $G$ can be achieved for a model in which the scalar field $\phi$ interacts with CDM through a momentum transfer [54 63. This type of interaction is based on the field derivative coupling with the CDM four velocity $u^{\mu}$ and it can be quantified by the scalar combination $Z=u^{\mu} \nabla_{\mu} \phi$, where $\nabla_{\mu}$ is the covariant derivative operator. In previous works, it was shown that the interacting Lagrangian such as $L_{\text {int }} \propto Z^{2}$ [54, 59] or $L_{\text {int }} \propto X^{1-n / 2} Z^{n}$ [64], where $X=-\nabla^{\mu} \phi \nabla_{\mu} \phi / 2$ and $n>0$, can realize $G_{\text {eff }}<G$ for the theories without energy transfer (i.e., $Q=0$ for the notation used above).

In the Schutz-Sorkin action approach, it is straightforward to accommodate both energy and momentum couplings between perfect-fluid dark matter and scalar-field dark energy. For the CDM density $\rho_{m}$ that depends on its number density $n$, the interacting action may be expressed in the form,

$$
\mathcal{S}_{\mathrm{int}}=\int \mathrm{d}^{4} x \sqrt{-g}\left[-f_{1}(\phi, X, Z) \rho_{m}(n)+f_{2}(\phi, X, Z)\right],
$$

where $g$ is the determinant of metric tensor $g_{\mu \nu}$, and $f_{1}, f_{2}$ are functions of $\phi, X, Z$. The first and second terms in the square bracket of Eq. (1.1) characterize the energy and momentum transfers, respectively. Unlike the other models in which phenomenological coupling terms are added to the background equations by hand 65 81, the evolution of cosmological perturbations is unambiguously fixed in our interacting theory with the explicit action (1.1).

The preceding discussion makes it clear that we need both scaling $\phi \mathrm{MDE}$ and weak gravity to alleviate the $H_{0}$ and $\sigma_{8}$ tensions. As it will be demonstrated in this work, these requirements can be satisfied when both energy and momentum exchanges occur between dark energy and CDM. For this purpose, we first derive a general interacting Lagrangian for the existence of scaling solutions. For simplicity, we assume Einstein gravity with the scalar interacting action (1.1) and the Schutz-Sorkin action for CDM, while we leave baryons and radiation uncoupled. In this case, there is no difference for the propagation of gravitational waves in comparison to general relativity and no screening mechanism is needed. We note that the function $f_{2}$ in Eq. (1.1) also accommodates the scaling Lagrangian derived for k-essence with the functional dependence $f_{2}(\phi, X) 8284$.

After obtaining the general scaling Lagrangian, we consider a subclass of models with $\phi \mathrm{MDE}$ and show that it is possible to realize $G_{\text {eff }}<G$ at low redshifts even in the presence of both energy and momentum transfers. The hope is that such interacting models can really ease both the $H_{0}$ and $\sigma_{8}$ tensions. We leave however the task of a full likelihood analysis with the current observational data for a future work.

\section{COUPLED DARK ENERGY WITH A LAGRANGIAN FORMULATION}

We consider a dark energy scalar field $\phi$ coupled to a barotropic perfect fluid described by a Schutz-Sorkin action [50, 51]. The interacting Lagrangian is generally given by the form $L(n, \phi, X, Z)$ [54, where $L$ is a function of the fluid number density $n$, the scalar field $\phi$ and its kinetic energy $X=-g^{\mu \nu} \nabla_{\mu} \phi \nabla_{\nu} \phi / 2$, and $Z=u^{\mu} \nabla_{\mu} \phi$, with $u^{\mu}$ being the fluid four velocity. We separate the interacting Lagrangian $L$ into the sums of energy transfer $-f_{1}(\phi, X, Z) \rho_{m}(n)$ and momentum transfer $f_{2}(\phi, X, Z)$, where $\rho_{m}$ is the fluid density that depends on $n$. For CDM, the density has the linear dependence $\rho_{m} \propto n$. For the gravity sector, we consider the Einstein-Hilbert action described by the Lagrangian $\left(M_{\mathrm{pl}}^{2} / 2\right) R$, where $R$ is the Ricci scalar. 
The total action is then given by

$$
\mathcal{S}=\int \mathrm{d}^{4} x \sqrt{-g} \frac{M_{\mathrm{pl}}^{2}}{2} R-\int \mathrm{d}^{4} x\left[\sqrt{-g} \rho_{m}(n)+J^{\mu} \partial_{\mu} \ell\right]+\int \mathrm{d}^{4} x \sqrt{-g} L(n, \phi, X, Z),
$$

where

$$
L(n, \phi, X, Z)=-f_{1}(\phi, X, Z) \rho_{m}(n)+f_{2}(\phi, X, Z) .
$$

The second integral in Eq. 2.1) corresponds to the Schutz-Sorkin action, where $n$ is related to the vector field $J^{\mu}$, as

$$
n=\sqrt{\frac{g^{\mu \nu} J_{\mu} J_{\nu}}{g}}
$$

Unlike Refs. [15, 16, we do not take vector perturbations into account in the Schutz-Sorkin action, as they are nondynamical in scalar-tensor theories. The fluid four-velocity $u_{\mu}$ is defined by

$$
u_{\mu} \equiv \frac{J_{\mu}}{n \sqrt{-g}}
$$

which satisfies the relation $u^{\mu} u_{\mu}=-1$. The scalar quantity $Z$ is expressed as $Z=g^{\mu \nu} J_{\mu} \nabla_{\nu} \phi /(n \sqrt{-g})$, while the scalar variable $\ell$ is a Lagrange multiplier. Since we are not modifying the Einstein-Hilbert action from general relativity, the speed of gravitational waves is equivalent to that of light.

Varying the action (2.1) with respect to $\ell$, it follows that

$$
\partial_{\mu} J^{\mu}=0
$$

The fluid pressure is given by [15, 16, 54, 56]

$$
P_{m}=n \rho_{m, n}-\rho_{m}
$$

Substituting $J^{\mu}=n \sqrt{-g} u^{\mu}$ into Eq. 2.5 and exploiting the properties $\partial_{\mu}\left(\sqrt{-g} u^{\mu}\right)=\sqrt{-g} \nabla_{\mu} u^{\mu}$ and $\left(\rho_{m}+P_{m}\right) \partial_{\mu} n=$ $n \partial_{\mu} \rho_{m}$, we obtain

$$
u^{\mu} \partial_{\mu} \rho_{m}+\left(\rho_{m}+P_{m}\right) \nabla_{\mu} u^{\mu}=0
$$

Variation of the action 2.1 with respect to $J^{\mu}$ leads to

$$
\partial_{\mu} \ell=u_{\mu}\left(1+f_{1}\right) \rho_{m, n}-\frac{f_{1, Z} \rho_{m}-f_{2, Z}}{n}\left(\nabla_{\mu} \phi+Z u_{\mu}\right)
$$

where we used the relation $\partial n / \partial J^{\mu}=J_{\mu} /(n g)$ and the notations $\rho_{m, n} \equiv \partial \rho_{m} / \partial n$ and $f_{i, Z} \equiv \partial f_{i} / \partial Z$ with $i=1,2$.

For the variation of action 2.1 with respect to $g^{\mu \nu}$, we employ the following properties,

$$
\begin{aligned}
\delta \sqrt{-g} & =-\frac{1}{2} \sqrt{-g} g_{\mu \nu} \delta g^{\mu \nu}, \\
\delta n & =\frac{n}{2}\left(g_{\mu \nu}-u_{\mu} u_{\nu}\right) \delta g^{\mu \nu}, \\
\delta X & =-\frac{1}{2} \nabla_{\mu} \phi \nabla_{\nu} \phi \delta g^{\mu \nu}, \\
\delta Z & =\left(\frac{1}{2} Z u_{\mu} u_{\nu}+u_{\mu} \nabla_{\nu} \phi\right) \delta g^{\mu \nu}, \\
\delta\left(J^{\mu} \partial_{\mu} \ell\right) & =\delta\left(g^{\mu \nu} J_{\mu} \partial_{\nu} \ell\right)=J_{\mu} \partial_{\nu} \ell \delta g^{\mu \nu} .
\end{aligned}
$$

When the action is varied with respect to $g^{\mu \nu}$, we need to keep $J^{\mu}$ fixed to reproduce the standard matter energymomentum tensor. Therefore, the vector $J^{\mu}$ should not be replaced with $n \sqrt{-g} u^{\mu}$ when varying the action. Then, we obtain the following covariant equations of motion,

$$
M_{\mathrm{pl}}^{2} G_{\mu \nu}=\left(1+f_{1}\right) T_{\mu \nu}^{(m)}+T_{\mu \nu}^{(\phi)}
$$


where $G_{\mu \nu}$ is the Einstein tensor, and

$$
\begin{aligned}
T_{\mu \nu}^{(m)} & =\left(\rho_{m}+P_{m}\right) u_{\mu} u_{\nu}+P_{m} g_{\mu \nu}, \\
T_{\mu \nu}^{(\phi)} & =f_{2} g_{\mu \nu}-\rho_{m}\left(f_{1, X} \nabla_{\mu} \phi \nabla_{\nu} \phi+f_{1, Z} Z u_{\mu} u_{\nu}\right)+f_{2, X} \nabla_{\mu} \phi \nabla_{\nu} \phi+f_{2, Z} Z u_{\mu} u_{\nu} .
\end{aligned}
$$

The matter energy-momentum tensor 2.15 satisfies the continuity equation,

$$
u^{\nu} \nabla^{\mu} T_{\mu \nu}^{(m)}=-\left[u_{\mu} \partial^{\mu} \rho_{m}+\left(\rho_{m}+P_{m}\right) \nabla^{\mu} u_{\mu}\right]=0,
$$

where we used Eq. (2.7) in the second equality. Taking the covariant derivative of Eq. 2.14), we obtain

$$
\left(1+f_{1}\right) \nabla^{\mu} T_{\mu \nu}^{(m)}+T_{\mu \nu}^{(m)} \nabla^{\mu} f_{1}+\nabla^{\mu} T_{\mu \nu}^{(\phi)}=0 .
$$

Multiplying Eq. (2.18) by $u^{\nu}$ and using the property (2.17), we find

$$
u^{\nu} \nabla^{\mu} T_{\mu \nu}^{(\phi)}=-u^{\nu} \nabla^{\mu} f_{1} T_{\mu \nu}^{(m)} .
$$

We define the effective matter energy-momentum tensor,

$$
\hat{T}_{\mu \nu}^{(m)} \equiv\left(1+f_{1}\right) T_{\mu \nu}^{(m)},
$$

which contains the effect of energy exchange between the scalar field and matter. This quantity obeys

$$
u^{\nu} \nabla^{\mu} \hat{T}_{\mu \nu}^{(m)}=+u^{\nu} \nabla^{\mu} f_{1} T_{\mu \nu}^{(m)} .
$$

The signs on the right hand sides of Eqs. (2.19) and 2.21) are opposite to each other, which shows the energy exchange between the scalar field and matter. The coupling $f_{2}$, which corresponds to the momentum transfer, does not appear on the right hand sides of Eqs. 2.19) and (2.21). However, the momentum transfer between the scalar field and matter occurs through Eq. (2.18).

Let us consider the flat Friedmann-Lemaitre-Robertson-Walker (FLRW) background given by the line element $\mathrm{d} s^{2}=-\mathrm{d} t^{2}+a^{2}(t) \delta_{i j} \mathrm{~d} x^{i} \mathrm{~d} x^{j}$, where $a(t)$ is the time-dependent scale factor. On this background we have $u^{\mu}=(1,0,0,0)$ and $\nabla_{\mu} u^{\mu}=3 H$, where $H=\dot{a} / a$ is the Hubble-Lemaitre expansion rate and a dot represents a derivative with respect to the cosmic time $t$. From Eq. (2.7), we obtain

$$
\dot{\rho}_{m}+3 H\left(\rho_{m}+P_{m}\right)=0,
$$

which corresponds to the conservation of total particle number $\mathcal{N} \equiv J^{0}=n a^{3}$. From the (00) and (ii) components of Einstein equations 2.14, it follows that

$$
\begin{aligned}
& 3 M_{\mathrm{pl}}^{2} H^{2}=\left(1+f_{1}\right) \rho_{m}+\rho_{\phi}, \\
& M_{\mathrm{pl}}^{2}\left(2 \dot{H}+3 H^{2}\right)=-\left(1+f_{1}\right) P_{m}-P_{\phi},
\end{aligned}
$$

where

$$
\begin{aligned}
& \rho_{\phi} \equiv-\rho_{m} f_{1, X} \dot{\phi}^{2}-\rho_{m} f_{1, Z} \dot{\phi}-f_{2}+f_{2, X} \dot{\phi}^{2}+f_{2, Z} \dot{\phi}, \\
& P_{\phi} \equiv f_{2} .
\end{aligned}
$$

From Eq. 2.20, we introduce the matter density and pressure containing the effect of energy transfer, as

$$
\hat{\rho}_{m} \equiv\left(1+f_{1}\right) \rho_{m}, \quad \hat{P}_{m} \equiv\left(1+f_{1}\right) P_{m} .
$$

From Eqs. 2.19) and 2.21, we have

$$
\begin{aligned}
& \dot{\rho}_{\phi}+3 H\left(\rho_{\phi}+P_{\phi}\right)=-\dot{f}_{1} \rho_{m}, \\
& \dot{\hat{\rho}}_{m}+3 H\left(\hat{\rho}_{m}+\hat{P}_{m}\right)=+\dot{f}_{1} \rho_{m},
\end{aligned}
$$

whose right hand sides explicitly show the energy exchange between the scalar field and matter.

For later convenience, we introduce the time-dependent density parameters,

$$
\Omega_{m} \equiv \frac{\left(1+f_{1}\right) \rho_{m}}{3 M_{\mathrm{pl}}^{2} H^{2}}, \quad \Omega_{\phi} \equiv \frac{\rho_{\phi}}{3 M_{\mathrm{pl}}^{2} H^{2}} .
$$


The form of matter is not specified until the end of Sec. IV, after which we take CDM, baryons, and radiation into account as perfect fluids. From Eq. 22.23), it follows that

$$
\Omega_{m}+\Omega_{\phi}=1
$$

We also define the equations of state,

$$
w_{m} \equiv \frac{\hat{P}_{m}}{\hat{\rho}_{m}}=\frac{P_{m}}{\rho_{m}}, \quad w_{\phi} \equiv \frac{P_{\phi}}{\rho_{\phi}},
$$

as well as the effective equation of state,

$$
w_{\text {eff }} \equiv w_{m} \Omega_{m}+w_{\phi} \Omega_{\phi}=-1-\frac{2 \dot{H}}{3 H^{2}} .
$$

The condition for cosmic acceleration to occur corresponds to $w_{\text {eff }}<-1 / 3$.

\section{SCALING LAGRANGIAN}

In this section, we derive the Lagrangian $L$ allowing for the existence of scaling solutions characterized by nonvanishing constants $\Omega_{m}$ and $\Omega_{\phi}$. Notice that we are not requiring that the entire cosmic evolution has the scaling behavior, but just that scaling epochs exist. Since $\left(1+f_{1}\right) \rho_{m} \propto H^{2}$ and $\rho_{\phi} \propto H^{2}$ in the scaling regime, it follows that

$$
\begin{aligned}
\frac{\dot{f}_{1}}{H\left(1+f_{1}\right)} & =-3\left(w_{\mathrm{eff}}-w_{m}\right), \\
\frac{\dot{\rho}_{\phi}}{H \rho_{\phi}} & =-3\left(1+w_{\mathrm{eff}}\right),
\end{aligned}
$$

where we used Eq. 2.22). We will consider the case in which $w_{\text {eff }}, w_{m}$, and $w_{\phi}$ are constant along the scaling solution. Then, all the terms in Eq. 2.24 are proportional to $H^{2}$. Since $\rho_{\phi} \propto P_{\phi} \propto H^{2}$, each term on the left hand side of Eq. 2.28 is proportional to $H^{3}$. On using Eq. (3.1), there is also the dependence $\dot{f}_{1} \rho_{m} \propto H\left(1+f_{1}\right) \rho_{m} \propto H^{3}$, so the scaling solution is consistent with Eq. 2.28) as well.

If we consider a canonical scalar field given by the Lagrangian $f_{2}(\phi, X)=X-V(\phi)$, the field density $\rho_{\phi}$ contains the kinetic energy $\dot{\phi}^{2} / 2$. In this case, the scaling solution satisfies the relation $\dot{\phi} \propto H$. To accommodate this model as a special case, we will derive the Lagrangian allowing for scaling solutions characterized by

$$
\frac{\dot{\phi}}{H M_{\mathrm{pl}}}=\alpha
$$

where $\alpha$ is a dimensionless constant. Then, the time derivative of $f_{1}(\phi, X, Z)$ yields

$$
\dot{f}_{1}=H\left[\alpha M_{\mathrm{pl}} f_{1, \phi}-3\left(1+w_{\mathrm{eff}}\right)\left(X f_{1, X}+\frac{1}{2} Z f_{1, Z}\right)\right] .
$$

Substituting Eq. (3.4) into Eq. (3.1), we obtain the partial differential equation,

$$
X f_{1, X}+\frac{1}{2} Z f_{1, Z}-\frac{1}{\lambda} M_{\mathrm{pl}} f_{1, \phi}+\frac{Q}{\lambda}\left(1+f_{1}\right)=0,
$$

where

$$
\lambda \equiv \frac{3\left(1+w_{\mathrm{eff}}\right)}{\alpha}, \quad Q \equiv-\frac{3\left(w_{\mathrm{eff}}-w_{m}\right)}{\alpha} .
$$

The integrated solution to Eq. 3.5 is given by

$$
f_{1}=e^{Q \phi / M_{\mathrm{pl}}} g_{1}\left(Y_{1}, Y_{2}\right)-1
$$

where $g_{1}$ is an arbitrary function of

$$
Y_{1}=X e^{\lambda \phi / M_{\mathrm{pl}}}, \quad Y_{2}=Z e^{\lambda \phi /\left(2 M_{\mathrm{pl}}\right)}
$$


Along the scaling solution, $\phi, a$, and $H$ evolve, respectively, as

$$
\phi=\phi_{0}+\alpha M_{\mathrm{pl}} \ln a, \quad a \propto\left(t-t_{0}\right)^{2 /\left[3\left(1+w_{\mathrm{eff}}\right)\right]}, \quad H=\frac{2}{3\left(1+w_{\mathrm{eff}}\right)\left(t-t_{0}\right)},
$$

where $\phi_{0}$ and $t_{0}$ are constants. This shows that both $Y_{1}$ and $Y_{2}$ remain constant in the scaling regime. One can also show that both $\rho_{m} f_{1, X} \dot{\phi}^{2}$ and $\rho_{m} f_{1, Z} \dot{\phi}$ in $\rho_{\phi}$ are proportional to $H^{2}$, so all the terms in Eq. 2.23 associated with the coupling $f_{1}$ obey the scaling relation. In other words, each term in $\rho_{\phi}$ arising from $f_{1}$ satisfies the same relation as Eq. (3.2).

The coupling $f_{2}$, which is equivalent to $P_{\phi}$ and is also present as one of the terms in $\rho_{\phi}$, should satisfy the scaling property $f_{2} \propto H^{2}$. This translates to the relation $\dot{f}_{2} /\left(H f_{2}\right)=-3\left(1+w_{\text {eff }}\right)$ and hence $f_{2}$ obeys the partial differential equation,

$$
X f_{2, X}+\frac{1}{2} Z f_{2, Z}-\frac{1}{\lambda} M_{\mathrm{pl}} f_{2, \phi}-f_{2}=0
$$

This is integrated to give

$$
f_{2}=X g_{2}\left(Y_{1}, Y_{2}\right),
$$

where $g_{2}$ is an arbitrary function of $Y_{1}$ and $Y_{2}$. On using the property that $Y_{1}$ and $Y_{2}$ are constant along the scaling solution, both $f_{2, X} \dot{\phi}^{2}$ and $f_{2, Z} \dot{\phi}$ in $\rho_{\phi}$ are proportional to $H^{2}$.

In summary, the Lagrangian allowing for the existence of scaling solutions is given by

$$
L=-\left[e^{Q \phi / M_{\mathrm{pl}}} g_{1}\left(Y_{1}, Y_{2}\right)-1\right] \rho_{m}(n)+X g_{2}\left(Y_{1}, Y_{2}\right),
$$

which contains arbitrary functions $g_{1}$ and $g_{2}$ with respect to $Y_{1}$ and $Y_{2}$. For the choice $g_{1}\left(Y_{1}, Y_{2}\right)=1$, the coupling $f_{1}$ corresponds to the one studied in Refs. [52, 62, i.e., $f_{1}=e^{Q \phi / M_{\mathrm{pl}}}-1$, where the constant $Q$ characterizes the strength of energy exchange. For k-essence theories with $f_{2}=f_{2}(\phi, X)$, the scaling Lagrangian reduces to $f_{2}=X g_{2}\left(Y_{1}\right)$, which agrees with that derived in Refs. 82 84. The $Z$ dependence in $f_{1}$ and $f_{2}$ gives rise to the additional $Y_{2}$ dependence in the functions $g_{1}$ and $g_{2}$.

\section{FIXED POINTS}

We derive the fixed points for interacting theories given by the action (2.1) with the scaling Lagrangian (3.12). In doing so, we introduce the following dimensionless variables,

$$
x \equiv \frac{\dot{\phi}}{\sqrt{6} H M_{\mathrm{pl}}}, \quad y \equiv \frac{M_{\mathrm{pl}} e^{-\lambda \phi /\left(2 M_{\mathrm{pl}}\right)}}{\sqrt{3} H} .
$$

Then, the background values of $Y_{1}$ and $Y_{2}$ are expressed, respectively, as

$$
Y_{1}=\frac{x^{2}}{y^{2}} M_{\mathrm{pl}}^{4}, \quad Y_{2}=\frac{\sqrt{2} x}{y} M_{\mathrm{pl}}^{2},
$$

and hence $Y_{1}=Y_{2}^{2} / 2$. It is also convenient to define

$$
\epsilon_{\phi} \equiv \frac{\ddot{\phi}}{H \dot{\phi}}, \quad \xi \equiv \frac{\dot{H}}{H^{2}} .
$$

Then, the variables $x$ and $y$ obey the differential equations,

$$
\begin{aligned}
& x^{\prime}=x\left(\epsilon_{\phi}-\xi\right), \\
& y^{\prime}=-y\left(\frac{\sqrt{6}}{2} \lambda x+\xi\right),
\end{aligned}
$$

where a prime denotes a derivative with respect to $N=\ln a$. On using Eqs. 22.23)-2.28, it follows that

$$
\begin{aligned}
\epsilon_{\phi} & =\frac{f_{1, \phi} \rho_{m}-f_{2, \phi}+3 H\left(f_{1, X} \dot{\phi}+f_{1, Z}\right) P_{m}+3 H\left(f_{2, X} \dot{\phi}+f_{2, Z}\right)-\left(f_{1, X \phi} \rho_{m}-f_{2, X \phi}\right) \dot{\phi}^{2}-\left(f_{1, Z \phi} \rho_{m}-f_{2, Z \phi}\right) \dot{\phi}}{\left[f_{1, X} \rho_{m}-f_{2, X}+\left(f_{1, X X} \rho_{m}-f_{2, X X}\right) \dot{\phi}^{2}+2\left(f_{1, X} \rho_{m}-f_{2, X}\right) \dot{\phi}+f_{1, Z Z} \rho_{m}-f_{2, Z Z}\right] H \dot{\phi}}, \\
\xi & =\frac{\left(f_{1, X} \dot{\phi}+f_{1, Z}\right) \dot{\phi} \rho_{m}-\left(f_{2, X} \dot{\phi}+f_{2, Z}\right) \dot{\phi}-\left(1+f_{1}\right)\left(\rho_{m}+P_{m}\right)}{2 H^{2} M_{\mathrm{pl}}^{2}} .
\end{aligned}
$$


For the theories given by the functions 3.7 and 3.11 , the quantities $\Omega_{\phi}, w_{\phi}$, and $w_{\text {eff }}$ are expressed as

$$
\begin{aligned}
\Omega_{\phi} & =\frac{g_{1} x^{2}\left(g_{2}+2 Y_{1} g_{2, Y_{1}}+Y_{2} g_{2, Y_{2}}\right)-2 Y_{1} g_{1, Y_{1}}-Y_{2} g_{1, Y_{2}}}{g_{1}-2 Y_{1} g_{1, Y_{1}}-Y_{2} g_{1, Y_{2}}}=1-\Omega_{m}, \\
w_{\phi} & =\frac{g_{2} x^{2}}{\Omega_{\phi}}, \\
w_{\text {eff }} & =g_{2} x^{2}+w_{m} \Omega_{m} .
\end{aligned}
$$

In Appendix, we will present the autonomous equations for the scaling Lagrangian 3.12 with $f_{1}$ and $f_{2}$ given by Eqs. (3.7) and (3.11), respectively.

The fixed points of the above dynamical system are characterized by constant values of $x$ and $y$, which we denote $x_{c}$ and $y_{c}$, respectively. The scaling fixed point corresponds to $x_{c} \neq 0$ and $y_{c} \neq 0$, so it should satisfy

$$
\begin{aligned}
& \epsilon_{\phi}=\xi, \\
& \xi=-\frac{\sqrt{6}}{2} \lambda x_{c} .
\end{aligned}
$$

From Eq. 4.12, we have

$$
g_{2, Y_{2}}=\frac{\sqrt{6} \lambda g_{1} x_{c}-3\left(1-\Omega_{\phi}\right)\left[\left(1+w_{m}\right) g_{1}-2 Y_{1} g_{1, Y_{1}}-Y_{2} g_{1, Y_{2}}\right]-6 g_{1} x_{c}^{2}\left(g_{2}+Y_{1} g_{2, Y_{1}}\right)}{3 g_{1} x_{c}^{2} Y_{2}} .
$$

Substituting this relation into Eq. 4.8 and solving it for $\Omega_{\phi}$, we obtain

$$
\Omega_{\phi}=1+\frac{3\left(1+g_{2} x_{c}^{2}\right)-\sqrt{6} \lambda x_{c}}{3 w_{m}} .
$$

By using Eqs. 4.11) and 4.13) with Eq. 4.8, it follows that

$$
\left(g_{1}-2 Y_{1} g_{1, Y_{1}}-Y_{2} g_{1, Y_{2}}\right)\left[2(Q+\lambda) x_{c}-\sqrt{6}\left(1+w_{m}\right)\right]\left[3\left(1+g_{2} x_{c}^{2}\right)-\sqrt{6} \lambda x_{c}\right]=0 .
$$

Provided that $g_{1}$ is different from the specific form $g_{1}=\sqrt{Y_{1}} F\left(Y_{2} / \sqrt{Y_{1}}\right)$, where $F$ is a function of $Y_{2} / \sqrt{Y_{1}}$, we have $g_{1}-2 Y_{1} g_{1, Y_{1}}-Y_{2} g_{1, Y_{2}} \neq 0$. Then, there are two fixed points satisfying Eq. 4.15): (a) scaling solution, and (b) scalar-field dominated point. In the following, we discuss the properties of them in turn.

\section{A. Scaling solution (a)}

The scaling fixed point corresponds to

$$
x_{c}=\frac{\sqrt{6}\left(1+w_{m}\right)}{2(Q+\lambda)} .
$$

Substituting Eq. 4.16) into Eqs. 4.14, 4.9), and 4.10), we obtain

$$
\begin{aligned}
\Omega_{\phi} & =\frac{\left[2 Q(Q+\lambda)+3\left(1+w_{m}\right) g_{2}\right]\left(1+w_{m}\right)}{2 w_{m}(Q+\lambda)^{2}}, \\
w_{\phi} & =\frac{3 w_{m}\left(1+w_{m}\right) g_{2}}{3\left(1+w_{m}\right) g_{2}+2 Q(Q+\lambda)}, \\
w_{\text {eff }} & =\frac{w_{m} \lambda-Q}{Q+\lambda} .
\end{aligned}
$$

Due to the constancy of $g_{2}\left(Y_{1}, Y_{2}\right)$ along the scaling solution, the quantities 4.17$)$ - 4.19$)$ do not vary in time. In the limit that $Q \rightarrow 0$, both $w_{\phi}$ and $w_{\text {eff }}$ reduce to $w_{m}$. In this case, the density of scalar field scales in the same manner as that of matter. The existence of nonvanishing coupling $Q$ leads $w_{\phi}$ and $w_{\text {eff }}$ being different from $w_{m}$.

Instead of using $g_{2}$ in Eq. (4.17), it is possible to express $\Omega_{\phi}$ in terms of $f_{2, X}, g_{2, Y_{2}}$ as well as $g_{1}$ and its derivatives with respect to $Y_{1}$ and $Y_{2}$. In doing so, we substitute Eq. (4.14) and the relation $g_{2, Y_{1}}=\left(f_{2, X}-g_{2}\right) / Y_{1}$ into Eq. (4.13) and solve it for $g_{2}$. Eliminating the term $g_{2}$ from Eq. 4.17), we obtain

$$
\Omega_{\phi}=\frac{\left[2 Q(Q+\lambda)+3\left(1+w_{m}\right)\left(2 f_{2, X}+Y_{2} g_{2, Y_{2}}\right)\right]\left(1+w_{m}\right) g_{1}-2(Q+\lambda)^{2}\left(2 Y_{1} g_{1, Y_{1}}+Y_{2} g_{1, Y_{2}}\right)}{2(Q+\lambda)^{2}\left[\left(1+w_{m}\right) g_{1}-2 Y_{1} g_{1, Y_{1}}-Y_{2} g_{1, Y_{2}}\right]} .
$$


If $g_{1}$ depends on neither $Y_{1}$ nor $Y_{2}$, we have $\Omega_{\phi}=\left[2 Q(Q+\lambda)+3\left(1+w_{m}\right)\left(2 f_{2, X}+Y_{2} g_{2, Y_{2}}\right)\right] /\left[2(Q+\lambda)^{2}\right]$. In k-essence where $g_{2}$ depends on $Y_{1}$ alone, the field density parameter further reduces to $\Omega_{\phi}=\left[Q(Q+\lambda)+3\left(1+w_{m}\right) f_{2, X}\right] /(Q+\lambda)^{2}$. This coincides with the result derived in Refs. [84, 85]. In quintessence $\left(f_{2}=X-V(\phi)\right)$ with the exponential potential $V(\phi)=V_{0} e^{-\lambda \phi / M_{\mathrm{pl}}}$, i.e., the choice $g_{2}=1-V_{0} / Y_{1}$, we reproduce the value $\Omega_{\phi}=3\left(1+w_{m}\right) / \lambda^{2}$ for $Q=0[86$. In this case, the scaling radiation and matter eras in which $\Omega_{\phi}$ is subdominant to $\Omega_{m}$ can be realized for $|\lambda| \gg 1$ [87, 88 .

The field density parameter 4.20 contains the dependence of both $Y_{1}$ and $Y_{2}$ in the functions $g_{1}$ and $g_{2}$, so it is the generalization of scaling solutions already known in literature. However, the effective equation of state (4.19) is not subject to modifications compared to that derived in Refs. [43, 84, 85. For $|\lambda| \gg 1$ and $|Q|<\mathcal{O}(1), w_{\text {eff }}$ is close to $w_{m}$. In this case, the scaling solution with $\Omega_{\phi} \ll 1$ can be used during the radiation or matter eras. For $|Q|$ larger than the order of $|\lambda|$, it is possible for the scaling solution to satisfy the condition of cosmic acceleration $\left(w_{\text {eff }}<-1 / 3\right)$. However, the coupling $|Q|$ is typically constrained to be smaller than 0.1 for the consistency with CMB measurements [45], in which case the realization of scaling accelerated attractor with $\Omega_{\phi} \simeq 0.7$ is difficult [84]. For $|Q|<\mathcal{O}(0.1)$ and $|\lambda|<\mathcal{O}(1)$, the fixed point relevant to late-time cosmic acceleration is the scalar-field dominated point discussed later in Sec. IVB.

\section{B. Scalar-field dominated point (b)}

From Eq. 4.15), there exists the other fixed point satisfying

$$
g_{2}=\frac{\sqrt{6} \lambda x_{c}-3}{3 x_{c}^{2}} .
$$

Then, from Eqs. 4.9, 4.10, and 4.14, we have

$$
\begin{aligned}
& \Omega_{\phi}=1, \\
& w_{\text {eff }}=w_{\phi}=-1+\frac{\sqrt{6} \lambda x_{c}}{3} .
\end{aligned}
$$

This scalar-field dominated point can be responsible for the late-time cosmic acceleration under the condition

$$
\lambda x_{c}<\frac{\sqrt{6}}{3} .
$$

From Eq. 4.13), there is also the following relation

$$
g_{2}+Y_{1} g_{2, Y_{1}}+\frac{1}{2} Y_{2} g_{2, Y_{2}}=\frac{\lambda}{\sqrt{6} x_{c}} .
$$

For a given function $g_{2}\left(Y_{1}, Y_{2}\right)$, the values of $x_{c}$ and $y_{c}$ at the scalar-field dominated point are obtained by solving Eqs. 4.21 and 4.25 together with the relations given in Eq. 4.2. On using Eq. 4.25), we can express Eq. 4.23) in the form

$$
w_{\text {eff }}=w_{\phi}=-1+\frac{2 \lambda^{2}}{3\left(2 f_{2, X}+Y_{2} g_{2, Y_{2}}\right)},
$$

where $f_{2, X}=g_{2}+Y_{1} g_{2, Y_{1}}$. In k-essence without the $Y_{2}$ dependence in $g_{2}$, we recover the value $w_{\text {eff }}=w_{\phi}=$ $-1+\lambda^{2} /\left(3 f_{2, X}\right)$ derived in Refs. [84, 85. From Eq. 4.26), we find that, for $\lambda$ closer to 0 , $w_{\text {eff }}$ and $w_{\phi}$ approach -1 .

\section{Kinetic fixed points}

Let us derive other fixed points for the dynamical system given by Eqs. 4.4 - 4.5 . The $\phi$ MDE corresponds to the scaling solution with kinetic domination satisfying

$$
y_{c}=0 \text {. }
$$

Since the quantities $Y_{1}$ and $Y_{2}$ diverge at $y_{c}=0$, the functions $g_{1}$ and $g_{2}$ should take the following forms to avoid the divergence of background equations,

$$
\begin{aligned}
& g_{1}\left(Y_{1}, Y_{2}\right)=b_{0}+\sum_{i>0}\left(b_{i} Y_{1}^{-i}+\tilde{b}_{i} Y_{2}^{-i}\right)+2^{1-m / 2} \mu \frac{Y_{2}^{m}}{Y_{1}^{m / 2}}+\sum_{i>0, j<2 i} \mu_{i} \frac{Y_{2}^{j}}{Y_{1}^{i}}, \\
& g_{2}\left(Y_{1}, Y_{2}\right)=c_{0}+\sum_{i>0}\left(c_{i} Y_{1}^{-i}+\tilde{c}_{i} Y_{2}^{-i}\right)+2^{1-m / 2} \beta \frac{Y_{2}^{m}}{Y_{1}^{m / 2}}+\sum_{i>0, j<2 i} \beta_{i} \frac{Y_{2}^{j}}{Y_{1}^{i}},
\end{aligned}
$$


where $b_{0}, b_{i}, \tilde{b}_{i}, \mu, \mu_{i}, c_{0}, c_{i}, \tilde{c}_{i}, \beta, \beta_{i}$, and $m$ are constants. Due to the relation $Y_{1}=Y_{2}^{2} / 2$ at the background level, the third terms on the right hand sides of Eqs. (4.28) and (4.29) are constant. The last terms in Eqs. (4.28) and (4.29) do not diverge for $i>0$ and $j<2 i$.

In the following, we will set $c_{0}=1$ without loss of generality. We substitute Eqs. (4.28)-(4.29) and their $Y_{1}, Y_{2}$ derivatives into Eq. 4.8)- (4.10), use the relations (4.2), and finally take the limit $y \rightarrow 0$. This process leads to

$$
\begin{aligned}
& \Omega_{\phi}=q_{s} x^{2}=1-\Omega_{m}, \\
& w_{\phi}=1, \\
& w_{\text {eff }}=w_{m}-\left(w_{m}-1\right) q_{s} x^{2},
\end{aligned}
$$

where

$$
q_{s} \equiv 1+2 \beta .
$$

The autonomous Eq. (4.4) reduces to

$$
x^{\prime}=-\frac{1}{2 q_{s}}\left[3 q_{s}\left(w_{m}-1\right) x-\sqrt{6} Q\right]\left(q_{s} x^{2}-1\right),
$$

while Eq. 4.5 is automatically satisfied. We note that the constants appearing in the expression of $g_{1}\left(Y_{1}, Y_{2}\right)$ in Eq. 4.28 do not affect Eq. 4.34). It is also interesting to observe that, for $w_{m}=0$, there is the relation $w_{\text {eff }}=\Omega_{\phi}=q_{s} x^{2}$. Indeed, this relation holds in all the kinetic scaling solutions identified so far, see, for instance, Refs. [52, 84, 89, 90]. It is this phase that induces a small and positive effective equation of state that can help alleviating the $H_{0}$ tension. On the other hand, this implies that a kinetic scaling solution associated with the matter dominance will be strongly constrained by $\mathrm{CMB}$ observations of the distance to last scattering. Leaving aside the $H_{0}$ tension, one possibility to implement $w_{\text {eff }}=0$, and therefore to ease CMB distance constraints, would be to introduce a non-vanishing $w_{m}=\Omega_{\phi} /\left(\Omega_{\phi}-1\right)$. This possibility is not pursued here but left for future work.

From Eq. 4.34, there are the following fixed points.

$$
\text { 1. } \phi M D E(\mathrm{c})
$$

The $\phi \mathrm{MDE}$ corresponds to one of the solutions to Eq. (4.34), i.e.,

$$
x_{c}=\frac{\sqrt{6} Q}{3 q_{s}\left(w_{m}-1\right)} .
$$

From Eqs. 4.30 and 4.32, we obtain

$$
\begin{aligned}
& \Omega_{\phi}=\frac{2 Q^{2}}{3 q_{s}\left(w_{m}-1\right)^{2}}=1-\Omega_{m}, \\
& w_{\text {eff }}=w_{m}-\frac{2 Q^{2}}{3 q_{s}\left(w_{m}-1\right)} .
\end{aligned}
$$

This means that the $\phi \mathrm{MDE}$ is a scaling solution besides the fixed point (a). For nonrelativistic matter $\left(w_{m}=0\right)$, it follows that $\Omega_{\phi}=w_{\text {eff }}=2 Q^{2} /\left(3 q_{s}\right)$. The standard matter-dominated epoch with $\Omega_{\phi}=w_{\text {eff }}=0$ is modified by the nonvanishing coupling $Q$. In comparison to theories with the coupling $Q$ alone, the coupling $\beta$ gives the additional contribution to $x_{c}, \Omega_{\phi}$, and $w_{\text {eff }}$.

\section{Purely kinetic solutions $(\mathrm{d} 1),(\mathrm{d} 2)$}

The other solution to Eq. 4.34 corresponds to purely kinetic points (d1), (d2) satisfying

$$
x_{c}= \pm \frac{1}{\sqrt{q_{s}}},
$$

whose existence requires that $q_{s}>0$. From Eqs. 4.30 and 4.32, we have

$$
\Omega_{\phi}=1, \quad w_{\text {eff }}=1 .
$$


The points (d1), (d2) are not relevant to radiation/matter eras or the epoch of cosmic acceleration.

In summary, the fixed points relevant to the cosmological evolution after radiation-matter equality are the $\phi \mathrm{MDE}$ (c) and the scalar-field dominated point (b). The coupling $Q$ associated with the energy transfer is crucially required for the existence of $\phi \mathrm{MDE}$. The coupling $\beta$ associated with the momentum transfer also affects the values of $\Omega_{\phi}$ and $w_{\text {eff }}$ on the $\phi \mathrm{MDE}$.

\section{BACKGROUND COSMOLOGY FOR A MODEL WITH $\phi$ MDE}

In this section, we study the background cosmological dynamics for the model with

$$
\begin{aligned}
& g_{1}\left(Y_{1}, Y_{2}\right)=1, \\
& g_{2}\left(Y_{1}, Y_{2}\right)=1-\frac{V_{0}}{Y_{1}}+2^{1-m / 2} \beta \frac{Y_{2}^{m}}{Y_{1}^{m / 2}},
\end{aligned}
$$

where $V_{0}$ is a positive constant. Although this is a simple model, it contains nevertheless all the new phenomenology we wish to consider. We consider the coupling only between the scalar field and CDM, where the CDM density $\rho_{c}$ depends on its number density $n_{c}$. The interacting model given by Eqs. (5.1)-(5.2) belongs to a subclass of the functions 4.28 and 4.29 , so the $\phi \mathrm{MDE}$ is present besides the other fixed points derived in Sec. IV] In this case, the Lagrangian (2.2) is given by

$$
L=-\left(e^{Q \phi / M_{\mathrm{p} 1}}-1\right) \rho_{c}\left(n_{c}\right)+X-V_{0} e^{-\lambda \phi / M_{\mathrm{pl}}}+\beta(2 X)^{1-m / 2} Z^{m} .
$$

The canonical scalar field $\phi$ with the potential $V(\phi)=V_{0} e^{-\lambda \phi / M_{\mathrm{pl}}}$ interacts with CDM through the energy-transfer $-\left(e^{Q \phi / M_{\mathrm{pl}}}-1\right) \rho_{c}\left(n_{c}\right)$ and the momentum-transfer $\beta(2 X)^{1-m / 2} Z^{m}$. Without the coupling $Q$, the interactions of the forms $Z^{2}$ [54, 59, 63] or $Z^{n}$ 61, 64] were already studied in the literature. In the following, we are going to investigate the cosmological dynamics in the presence of the two nonvanishing coupling constants $Q$ and $\beta$.

Besides CDM with the vanishing pressure $\left(P_{c}=0\right)$, we also take baryons (energy density $\rho_{b}$ with vanishing pressure) and radiation (energy density $\rho_{r}$ and pressure $P_{r}=\rho_{r} / 3$ ) into account to study the dynamics of background cosmology from the radiation era. Neither baryons nor radiation are assumed to be coupled to the scalar field.

Defining the variable $x$ as in Eq. 4.1), the dimensionless scalar field $\tilde{\phi} \equiv \phi / M_{\mathrm{pl}}$ obeys the differential equation,

$$
\tilde{\phi}^{\prime}=\sqrt{6} x .
$$

Instead of the variable $y$ defined in Eq. 4.1, we will use

$$
\tilde{y} \equiv \frac{\sqrt{V_{0}}}{M_{\mathrm{pl}}^{2}} y=\sqrt{\frac{V_{0}}{3}} \frac{e^{-\lambda \phi /\left(2 M_{\mathrm{pl}}\right)}}{M_{\mathrm{pl}} H} .
$$

The density parameters of scalar field and matter components are given by

$$
\Omega_{\phi}=q_{s} x^{2}+\tilde{y}^{2}, \quad \Omega_{c}=\frac{e^{Q \phi / M_{\mathrm{pl}}} \rho_{c}}{3 M_{\mathrm{pl}}^{2} H^{2}}, \quad \Omega_{b}=\frac{\rho_{b}}{3 M_{\mathrm{pl}}^{2} H^{2}}, \quad \Omega_{r}=\frac{\rho_{r}}{3 M_{\mathrm{pl}}^{2} H^{2}} .
$$

As we will see in Sec. VI the no-ghost condition of scalar-field perturbation requires that

$$
q_{s}=1+2 \beta>0 .
$$

From Eq. 2.23), 2.24 and 2.28), we have

$$
\begin{aligned}
\Omega_{c} & =1-\Omega_{\phi}-\Omega_{b}-\Omega_{r}, \\
\xi & =-3 q_{s} x^{2}-\frac{3}{2} \Omega_{c}-\frac{3}{2} \Omega_{b}-2 \Omega_{r}, \\
\epsilon_{\phi} & =-3+\frac{\sqrt{6}}{2 q_{s} x}\left(\lambda \tilde{y}^{2}-Q \Omega_{c}\right),
\end{aligned}
$$


where we recall that $\xi$ and $\epsilon_{\phi}$ are defined in Eq. (4.3). We can reduce the background equations to the following autonomous system,

$$
\begin{aligned}
x^{\prime} & =\frac{1}{2} x\left(6 q_{s} x^{2}-6+3 \Omega_{c}+3 \Omega_{b}+4 \Omega_{r}\right)+\frac{\sqrt{6}}{2 q_{s}}\left(\lambda \tilde{y}^{2}-Q \Omega_{c}\right), \\
\tilde{y}^{\prime} & =\frac{1}{2} \tilde{y}\left(6 q_{s} x^{2}-\sqrt{6} \lambda x+3 \Omega_{c}+3 \Omega_{b}+4 \Omega_{r}\right), \\
\Omega_{b}^{\prime} & =\Omega_{b}\left(6 q_{s} x^{2}-3+3 \Omega_{c}+3 \Omega_{b}+4 \Omega_{r}\right), \\
\Omega_{r}^{\prime} & =\Omega_{r}\left(6 q_{s} x^{2}-4+3 \Omega_{c}+3 \Omega_{b}+4 \Omega_{r}\right) .
\end{aligned}
$$

Notice that the background equations are independent of the power $m$. The scalar-field and effective equations of state are given, respectively, by

$$
\begin{aligned}
w_{\phi} & =\frac{q_{s} x^{2}-\tilde{y}^{2}}{q_{s} x^{2}+\tilde{y}^{2}}, \\
w_{\mathrm{eff}} & =-1+2 q_{s} x^{2}+\Omega_{c}+\Omega_{b}+\frac{4}{3} \Omega_{r} .
\end{aligned}
$$

Besides the scaling fixed point (a) derived in Sec. IV there exist the following three fixed points (b), (c), and (e) which are relevant to the dynamics of accelerated, matter, and radiation eras, respectively.

- Accelerated point (b)

$$
x_{c}=\frac{\lambda}{\sqrt{6} q_{s}}, \quad \tilde{y}_{c}=\sqrt{1-\frac{\lambda^{2}}{6 q_{s}}}, \quad \Omega_{b}=0, \quad \Omega_{r}=0, \quad \Omega_{\phi}=1, \quad w_{\phi}=w_{\mathrm{eff}}=-1+\frac{\lambda^{2}}{3 q_{s}} .
$$

- $\phi \mathrm{MDE}$ point (c)

$$
x_{c}=-\frac{\sqrt{6} Q}{3 q_{s}}, \quad \tilde{y}_{c}=0, \quad \Omega_{b}=0, \quad \Omega_{r}=0, \quad \Omega_{\phi}=w_{\mathrm{eff}}=\frac{2 Q^{2}}{3 q_{s}}, \quad w_{\phi}=1 .
$$

- Radiation point (e)

$$
x_{c}=0, \quad \tilde{y}_{c}=0, \quad \Omega_{b}=0, \quad \Omega_{r}=1, \quad \Omega_{\phi}=0, \quad w_{\mathrm{eff}}=\frac{1}{3} .
$$

The point (b) can drive a late-time cosmic acceleration under the condition $w_{\text {eff }}<-1 / 3$. This translates to

$$
\lambda^{2}<2 q_{s}
$$

From Eq. 4.19 , there is a possibility that the scaling solution (a) leads to the acceleration for $|Q| \gg|\lambda|$. As shown in Ref. 84], however, such a large coupling $|Q|$ is hardly compatible with the existence of $\phi \mathrm{MDE}(\mathrm{c})$ with $\Omega_{\phi} \ll 1$. As we will also see below, if point (b) is stable, then point (a) is not. Hence we will focus on the case in which $\phi$ MDE is followed by point (b).

Besides point (e), there exist the scaling fixed points (f) and (g) given by

$$
\begin{aligned}
& \text { (f) } x_{c}=\frac{2 \sqrt{6}}{3 \lambda}, \quad \tilde{y}_{c}=\sqrt{\frac{4 q_{s}}{3 \lambda^{2}}}, \quad \Omega_{b}=0, \quad \Omega_{r}=1-\frac{4 q_{s}}{\lambda^{2}}, \quad \Omega_{\phi}=\frac{4 q_{s}}{\lambda^{2}}, \quad w_{\phi}=w_{\mathrm{eff}}=\frac{1}{3}, \\
& \text { (g) } x_{c}=-\frac{1}{\sqrt{6} Q}, \quad \tilde{y}_{c}=0, \quad \Omega_{b}=0, \quad \Omega_{r}=1-\frac{q_{s}}{2 Q^{2}}, \quad \Omega_{\phi}=\frac{q_{s}}{6 Q^{2}}, \quad w_{\phi}=1, \quad w_{\mathrm{eff}}=\frac{1}{3},
\end{aligned}
$$

both of which can be potentially used for the radiation era. For point (f), however, $\Omega_{r}$ is negative under the condition 5.20. The point (g) can be responsible for the radiation era only for $Q^{2} \gg q_{s}$, which was exploited for the generation of primordial dark matter halos in Ref. 91. Unless some screening of fifth forces occurs after radiation-matter equality, point (g) is not followed by $\phi \mathrm{MDE}$ with $\Omega_{\phi} \ll 1$. Hence we use neither (f) nor (g) for the fixed point of radiation era in this paper. In other words, we consider the cosmological sequence of fixed points: (e) $\rightarrow(\mathrm{c}) \rightarrow(\mathrm{b})$.

The stability of fixed points is established by perturbing Eqs. (5.11)-(5.14) with the linear perturbations $\delta x, \delta \tilde{y}$, $\delta \Omega_{b}$, and $\delta \Omega_{r}$. The signs of eigenvalues of $4 \times 4$ Jacobian matrices for these perturbations determine whether the fixed points are stable or not [86, 88]. For points (e) and (c), some of the eigenvalues are positive, so they are not stable 
nodes. In other words, the $\phi \operatorname{MDE}(\mathrm{c})$, which is preceded by point (e), should eventually come to end to realize cosmic acceleration. Provided that the condition $(5.20)$ is satisfied, three of the eigenvalues for point (b) are negative. The other eigenvalue is negative for

$$
\lambda(\lambda+Q)<3 q_{s}
$$

under which point (b) is stable. On the other hand, the stability of point (a) requires that $\lambda(\lambda+Q)>3 q_{s}$, which is opposite to the inequality (5.23). This means that, under the condition (5.23), the $\phi$ MDE is followed by point (b) instead of point (a). We show a phase-space plot in Fig. 3 of Appendix to confirm the attractor property of point (b).

In Fig. 1. we plot the evolution of $\Omega_{\phi}, \Omega_{c}, \Omega_{b}$, and $\Omega_{r}$ (left) and $x, \tilde{y}, w_{\phi}$, and $w_{\text {eff }}$ (right) for the model parameters $\lambda=1, Q=0.07$, and $\beta=0.5$. We observe that the radiation fixed point (e) is followed by the $\phi \operatorname{MDE}$ (c) with nearly constant values $\Omega_{\phi}=w_{\text {eff }} \simeq 2 Q^{2} /\left(3 q_{s}\right)$. The field equation of state $w_{\phi}$ during the $\phi \mathrm{MDE}$ is close to 1 , whose property is attributed to the kinetically driven evolution satisfying $|x| \simeq \sqrt{6}|Q| /\left(3 q_{s}\right) \gg \tilde{y}$.

Since the model parameters $\lambda$ and $\beta$ used in Fig. 1 satisfy the two conditions $(5.20)$ and $(5.23)$, the solutions finally approach the stable accelerated point (b) with the asymptotic values $x=0.204, \tilde{y}=0.957$, and $w_{\phi}=w_{\text {eff }}=-0.833$. They are in good agreement with the numerical results shown in the right panel. In this case, we observe that $w_{\phi}$ temporarily reaches the minimum around -1 at redshift $z \simeq 1.3$ and then it increases toward the asymptotic value -0.833 . This evolution of $w_{\phi}$ is different from that for the model with $Q=0$ where $w_{\phi}$ is close to -1 during the matter era and finally approaches $-1+\lambda^{2} /\left(3 q_{s}\right)$ [64. Thus, the two models can be observationally distinguished from each other.
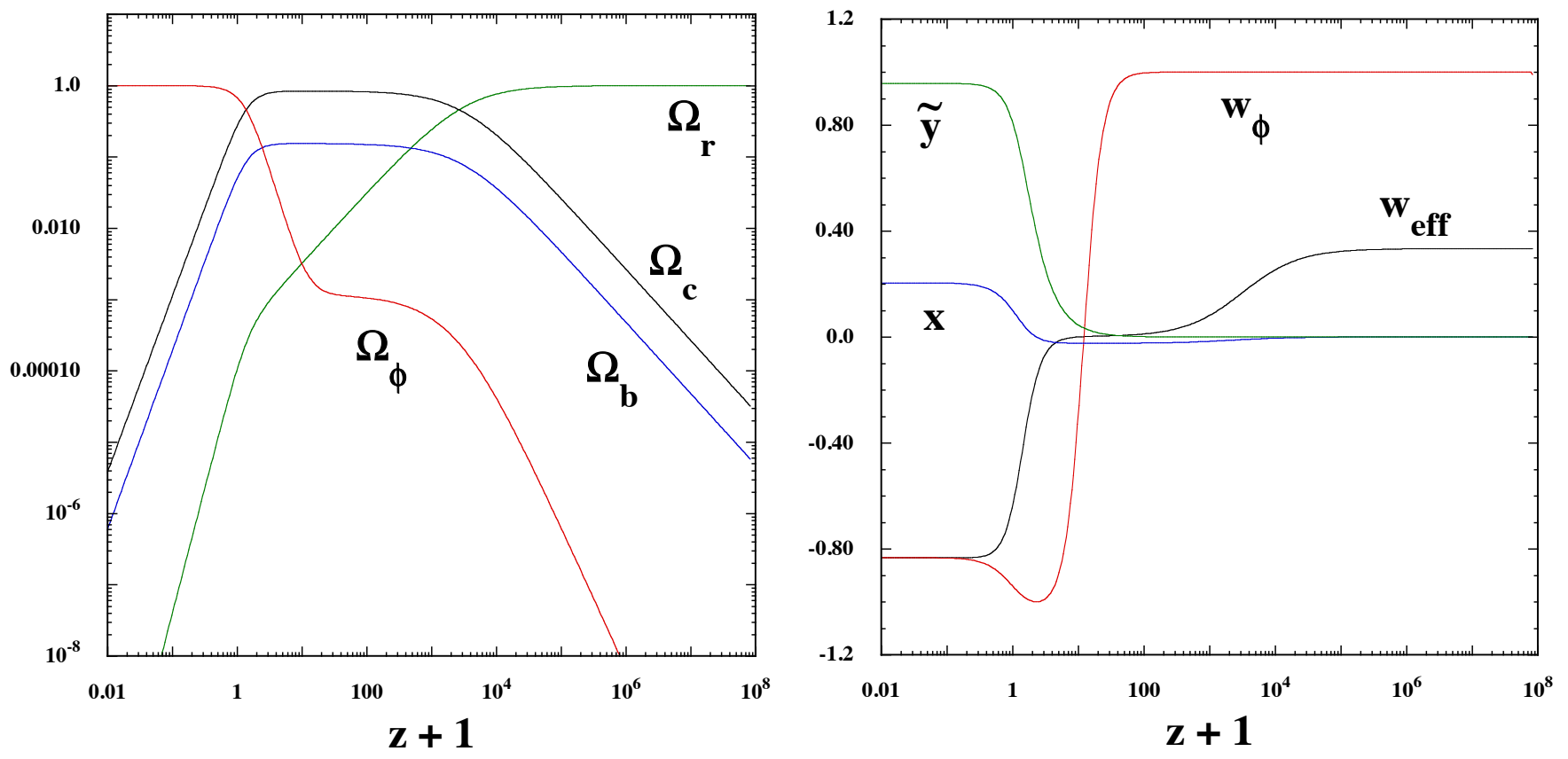

FIG. 1. (Left) Evolution of $\Omega_{\phi}, \Omega_{c}, \Omega_{b}$, and $\Omega_{r}$ versus $z+1$ for $\lambda=1, Q=0.07$, and $\beta=0.5$, where $z=a_{0} / a-1$ and $a_{0}$ is today's value of $a$. The initial conditions are chosen to be $x=1.0 \times 10^{-13}, \tilde{y}=1.0 \times 10^{-14}, \Omega_{b}=5.8 \times 10^{-6}$, and $\Omega_{r}=0.999962$ at redshift $z=8.3 \times 10^{7}$. (Right) Evolution of $x, \tilde{y}, w_{\phi}$, and $w_{\text {eff }}$ for the same model parameters and initial conditions as those used in the left.

We note that the values of $\Omega_{\phi}$ and $w_{\text {eff }}$ on the $\phi \mathrm{MDE}$ are reduced by the positive coupling $\beta$. The presence of positive $\beta$ also leads to the decreases of $w_{\phi}$ and $w_{\text {eff }}$ on the attractor point (b). For increasing $\beta$, the asymptotic value of $w_{\phi}$ gets closer to -1 . Nevertheless, the early evolution of $w_{\phi}$ is different from that in the $\Lambda$ CDM model. It remains to be seen whether the problem of $H_{0}$ tension in the $\Lambda$ CDM model can be alleviated in our interacting model with $Q \neq 0$ and $\beta \neq 0$. 


\section{PERTURBATION DYNAMICS FOR A MODEL WITH $\phi$ MDE}

In this section, we study the evolution of linear scalar perturbations for the theory 2.1 with the interacting Lagrangian (5.3). On the flat FLRW background, we consider the perturbed line element given by

$$
\mathrm{d} s^{2}=-(1+2 \alpha) \mathrm{d} t^{2}+2 \partial_{i} \chi \mathrm{d} t \mathrm{~d} x^{i}+a^{2}(t)\left[(1+2 \zeta) \delta_{i j}+2 \partial_{i} \partial_{j} E\right] \mathrm{d} x^{i} \mathrm{~d} x^{j} .
$$

The quantities $\alpha, \chi, \zeta$, and $E$ are scalar perturbations, which depend on both $t$ and spatial coordinate $x^{i}$. Here and in the following, we use the notation $\partial_{i} \chi=\partial \chi / \partial x^{i}$. We do not take the tensor perturbation into account in Eq. 6.1, but it propagates in the same manner as in standard general relativity. We decompose the scalar field $\phi$ into the background part $\bar{\phi}(t)$ and the perturbation $\delta \phi$, as

$$
\phi=\bar{\phi}(t)+\delta \phi\left(t, x^{i}\right),
$$

where we omit the bar from background quantities in the following.

For perfect fluids, we take CDM, baryons, and radiation into account labelled by $I=c, b, r$, respectively. From Eq. 2.5, it follows that the number $\mathcal{N}_{I}=n_{I} a^{3}$ of each fluid is conserved at the background level. We express the temporal and spatial components of $J_{I}^{\mu}$, respectively, as,

$$
J_{I}^{0}=\mathcal{N}_{I}+\delta J_{I}, \quad J_{I}^{i}=\frac{1}{a^{2}(t)} \delta^{i k} \partial_{k} \delta j_{I},
$$

where $\delta J_{I}$ and $\delta j_{I}$ are the scalar perturbations. The scalar velocity potential $v_{I}$ is related to the spatial component of four velocity $u_{I i}=J_{I i} /\left(n_{I} \sqrt{-g}\right)$, as

$$
u_{I i}=-\partial_{i} v_{I} .
$$

Since $J_{I i}=J_{I}^{0} g_{0 i}+J_{I}^{j} g_{i j}=\mathcal{N}_{I} \partial_{i} \chi+\partial_{i} \delta j_{I}$ at linear order in perturbations, it follows that

$$
\partial_{i} \delta j_{I}=-\mathcal{N}_{I}\left(\partial_{i} \chi+\partial_{i} v_{I}\right) .
$$

On using Eq. 2.8, there is also the following relation

$$
\ell_{c}=-\int^{t}\left[1+f_{1}(\tilde{t})\right] \rho_{c, n_{c}}(\tilde{t}) \mathrm{d} \tilde{t}-\left(1+f_{1}\right) \rho_{c, n_{c}} v_{c}+\frac{f_{1, Z} \rho_{c}-f_{2, Z}}{n_{c}}\left(\dot{\phi} v_{c}-\delta \phi\right),
$$

up to first order in perturbations. On the other hand, the Lagrange multipliers $\ell_{I}$ for baryons and radiation are

$$
\ell_{I}=-\int^{t} \rho_{I, n_{I}}(\tilde{t}) \mathrm{d} \tilde{t}-\rho_{I, n_{I}} v_{I}, \quad \text { with } I=b, r .
$$

The fluid density is given by $\rho_{I}=\rho_{I}(t)+\delta \rho_{I}\left(t, x^{i}\right)$, where the perturbed part is

$$
\delta \rho_{I}=\frac{\rho_{I, n_{I}}}{a^{3}}\left[\delta J_{I}-\mathcal{N}_{I}\left(3 \zeta+\partial^{2} E\right)\right],
$$

where $\partial^{2} E=\sum_{i=1}^{3} \partial_{i}^{2} E$. In this case, we have

$$
\delta n_{I}=\frac{\delta \rho_{I}}{\rho_{I, n_{I}}}-\frac{\mathcal{N}_{I}\left(\partial v_{I}\right)^{2}}{2 a^{5}}-\left(3 \zeta+\partial^{2} E\right) \frac{\delta \rho_{I}}{\rho_{I, n_{I}}}-\frac{\mathcal{N}_{I}\left(\zeta+\partial^{2} E\right)\left(3 \zeta-\partial^{2} E\right)}{2 a^{3}}+\mathcal{O}\left(\varepsilon^{3}\right),
$$

where $\left(\partial v_{I}\right)^{2}=\sum_{i=1}^{3}\left(\partial_{i} v_{I}\right)^{2}$, and $\varepsilon^{n}$ represents the $n$-th order of perturbations. We also introduce the sound speed squared of each matter fluid, as

$$
c_{I}^{2}=\frac{n_{I} \rho_{I, n_{I} n_{I}}}{\rho_{I, n_{I}}} .
$$

The quantities $\rho_{I}\left(n_{I}\right), X$, and $Z$, when are expanded up to second order in perturbations, are given by

$$
\begin{aligned}
\rho_{I}\left(n_{I}\right) & =\rho_{I}+\left(\rho_{I}+P_{I}\right) \frac{\delta n_{I}}{n_{I}}+\frac{1}{2}\left(\rho_{I}+P_{I}\right) c_{I}^{2}\left(\frac{\delta n_{I}}{n_{I}}\right)^{2}+\mathcal{O}\left(\varepsilon^{3}\right), \\
X & =\frac{1}{2} \dot{\phi}^{2}+\dot{\phi}(\dot{\delta \phi}-\dot{\phi} \alpha)+\frac{1}{2}\left[(\dot{\delta \phi}-2 \dot{\phi} \alpha)^{2}-\frac{1}{a^{2}}(\partial \delta \phi+\dot{\phi} \partial \chi)^{2}\right]+\mathcal{O}\left(\varepsilon^{3}\right), \\
Z & =\dot{\phi}+\dot{\delta \phi}-\dot{\phi} \alpha+\frac{1}{2 a^{2}}\left[\dot{\phi}\left\{3 a^{2} \alpha^{2}-\left(\partial_{i} \chi\right)^{2}+\left(\partial_{i} v_{c}\right)^{2}\right\}-2 a^{2} \alpha \dot{\phi \phi}-2 \partial_{i} \delta \phi\left(\partial_{i} \chi+\partial_{i} v_{c}\right)\right]+\mathcal{O}\left(\varepsilon^{3}\right) .
\end{aligned}
$$


In the following, we consider the case in which the equations of state and sound speed squares of both CDM and baryons vanish, i.e.,

$$
w_{c}^{2}=0, \quad w_{b}^{2}=0, \quad c_{c}^{2}=0, \quad c_{b}^{2}=0 .
$$

As in Refs. 62, 64, the linear perturbation equations of motion follow by expanding the action (2.1) up to quadratic order. Alternatively, we can also derive them by using the covariant equations of motion (2.14), (2.17), and (2.18) at first order. The explicit form of second-order action will be presented for a more general interacting Lagrangian $L\left(n_{c}, \phi, X, Z\right)$ as a future work 92. Here, we show the perturbation equations for the interacting Lagrangian (5.3) in a gauge-ready form. For this purpose, we introduce the following gauge-invariant quantities,

$$
\begin{aligned}
& \delta \phi_{\mathrm{N}}=\delta \phi+\dot{\phi}\left(\chi-a^{2} \dot{E}\right), \quad \delta \rho_{I \mathrm{~N}}=\delta \rho_{I}+\dot{\rho}_{I}\left(\chi-a^{2} \dot{E}\right), \quad v_{I \mathrm{~N}}=v_{I}+\chi-a^{2} \dot{E}, \\
& \Psi=\alpha+\frac{\mathrm{d}}{\mathrm{d} t}\left(\chi-a^{2} \dot{E}\right), \quad \Phi=\zeta+H\left(\chi-a^{2} \dot{E}\right),
\end{aligned}
$$

together with the dimensionless variables,

$$
\delta_{I \mathrm{~N}}=\frac{\delta \rho_{I \mathrm{~N}}}{\rho_{I}}, \quad \delta \varphi_{\mathrm{N}}=\frac{H}{\dot{\phi}} \delta \phi_{\mathrm{N}}, \quad V_{I \mathrm{~N}}=H v_{I \mathrm{~N}}, \quad \mathcal{K}=\frac{k}{a H},
$$

where $k$ is a comoving wavenumber.

In Fourier space, the full set of linear perturbation equations of motion is then given by

$$
\begin{aligned}
& 6 q_{s} x^{2} \delta \varphi_{\mathrm{N}}^{\prime}-6 \Phi^{\prime}+6\left(1-q_{s} x^{2}\right)\left(\xi \delta \varphi_{\mathrm{N}}+\Psi\right)-2 \mathcal{K}^{2} \Phi+3\left(3 \Omega_{c}+3 \Omega_{b}+4 \Omega_{r}\right) \delta \varphi_{\mathrm{N}} \\
& +3\left(\Omega_{c} \delta_{c \mathrm{~N}}+\Omega_{b} \delta_{b \mathrm{~N}}+\Omega_{r} \delta_{r \mathrm{~N}}\right)=0, \\
& \Phi^{\prime}-\Psi-\xi \delta \varphi_{\mathrm{N}}+\frac{3}{2}\left(\Omega_{c}+2 m \beta x^{2}\right)\left(V_{c \mathrm{~N}}-\delta \varphi_{\mathrm{N}}\right)+\frac{3}{2} \Omega_{b}\left(V_{b \mathrm{~N}}-\delta \varphi_{\mathrm{N}}\right) \\
& +2 \Omega_{r}\left(V_{r \mathrm{~N}}-\delta \varphi_{\mathrm{N}}\right)=0, \\
& \delta_{I \mathrm{~N}}^{\prime}+3\left(c_{I}^{2}-w_{I}\right) \delta_{I \mathrm{~N}}+\left(1+w_{I}\right)\left(\mathcal{K}^{2} V_{I \mathrm{~N}}+3 \Phi^{\prime}\right)=0, \quad(\text { for } I=c, b, r), \\
& \left(\Omega_{c}+2 m \beta x^{2}\right) V_{c \mathrm{~N}}^{\prime}-\left[\xi\left(\Omega_{c}+2 m \beta x^{2}\right)-2 m \beta x^{2}\left(3+2 \epsilon_{\phi}\right)-\sqrt{6} Q x \Omega_{c}\right] V_{c \mathrm{~N}}-\Omega_{c} \Psi \\
& -2 m \beta x^{2} \delta \varphi_{\mathrm{N}}^{\prime}+\left[2 m \beta x\left(\xi-3-2 \epsilon_{\phi}\right)-\sqrt{6} Q \Omega_{c}\right] x \delta \varphi_{\mathrm{N}}=0, \\
& V_{I \mathrm{~N}}^{\prime}-\left(\xi+3 c_{I}^{2}\right) V_{I \mathrm{~N}}-\Psi-\frac{c_{I}^{2}}{1+w_{I}} \delta_{I \mathrm{~N}}=0, \quad(\text { for } I=b, r), \\
& \delta \varphi_{\mathrm{N}}^{\prime \prime}+\left(3-\xi+2 \epsilon_{\phi}\right) \delta \varphi_{\mathrm{N}}^{\prime}+\left[\hat{c}_{s}^{2} \mathcal{K}^{2}-\xi^{\prime}-3 \xi+\epsilon_{\phi}^{\prime}+\epsilon_{\phi}^{2}+(3-\xi) \epsilon_{\phi}+\frac{3}{q_{s}}\left(\lambda^{2} \tilde{y}^{2}+Q^{2} \Omega_{c}\right)\right] \delta \varphi_{\mathrm{N}} \\
& +3 \hat{c}_{s}^{2} \Phi^{\prime}-\Psi^{\prime}-2\left(3+\epsilon_{\phi}\right) \Psi-\frac{m \beta}{q_{s}} \delta_{c \mathrm{~N}}^{\prime}+\frac{\sqrt{6} Q \Omega_{c}}{2 q_{s} x} \delta_{c \mathrm{~N}}=0, \\
& \Psi=-\Phi,
\end{aligned}
$$

where we remind that the prime denotes the derivative with respect to $N=\ln a$, and

$$
\hat{c}_{s}^{2}=1-\frac{m \beta}{q_{s}} .
$$

Equations (6.17)-(6.23) are written in the gauge-ready form, in that they can be used for any gauge choices of interest. For example, if we choose the unitary gauge characterized by $\delta \phi=0$ and $E=0$, the dynamical perturbations correspond to the curvature perturbation $\mathcal{R}=\Phi-\delta \varphi_{\mathrm{N}}$ and the density perturbations $\delta \rho_{I \mathrm{u}}=\delta \rho_{I \mathrm{~N}}-\dot{\rho}_{I} \delta \phi_{\mathrm{N}} / \dot{\phi}$. We can eliminate nondynamical variables like $\alpha, \chi$, and $v_{I}$ from the second-order action by using Eqs. (6.17)-(6.19). Following a procedure similar to that performed in Refs. 62, 64 for perturbations deep inside the Hubble radius, there are neither ghosts nor Laplacian instabilities for dynamical perturbations $\mathcal{R}$ and $\delta \rho_{c u}$ under the conditions,

$$
\begin{aligned}
q_{s} & =1+2 \beta>0, \\
q_{c} & =1+\frac{2 m \beta x^{2}}{\Omega_{c}}>0, \\
c_{s}^{2} & =\hat{c}_{s}^{2}+\frac{2 \beta^{2} m^{2} x^{2}}{q_{s}\left(2 \beta m x^{2}+\Omega_{c}\right)} \geq 0 .
\end{aligned}
$$


In the limit that $c_{c}^{2} \rightarrow 0$, the effective sound speed squared of CDM vanishes, so that there is no additional pressure modifying the evolution of CDM density perturbations. The effective sound speed squared of the scalar field in the small-scale limit corresponds to $c_{s}^{2}$ given in Eq. 6.27). In comparison to the value $\hat{c}_{s}^{2}$, there is a correction to $c_{s}^{2}$ arising from a kinetic mixing between the scalar field and CDM 62, 64. This correction term is positive under the no-ghost conditions (6.25) and (6.26). Provided that $\hat{c}_{s}^{2} \geq 0$, the positivity of $c_{s}^{2}$ is always ensured.

In the following, we study the evolution of perturbations after the onset of $\phi \mathrm{MDE}$. For this purpose, we ignore the contribution of radiation perturbations to Eqs. 66.17)- 6.23) and set $\Omega_{r}=0$. For CDM and baryons, Eq. 6.19) reduces to

$$
\delta_{I \mathrm{~N}}^{\prime}+\mathcal{K}^{2} V_{I \mathrm{~N}}+3 \Phi^{\prime}=0,
$$

where $I=c, b$. The CDM velocity potential $V_{c \mathrm{~N}}$ satisfies the first-order differential Eq. 6.20), while the baryon velocity potential $V_{b \mathrm{~N}}$ obeys

$$
V_{b \mathrm{~N}}^{\prime}-\xi V_{b \mathrm{~N}}-\Psi=0 .
$$

Differentiating Eq. (6.28) with respect to $N$ and using Eqs. 6.20) and 6.29), it follows that

$$
\begin{aligned}
& \delta_{c \mathrm{~N}}^{\prime \prime}+\nu_{1} \delta_{c \mathrm{~N}}^{\prime}+\nu_{2} \mathcal{K}^{2}+3 \Phi^{\prime \prime}+3 \nu_{1} \Phi^{\prime}=0, \\
& \delta_{b \mathrm{~N}}^{\prime \prime}+(2+\xi) \delta_{b \mathrm{~N}}^{\prime}+\mathcal{K}^{2} \Psi+3 \Phi^{\prime \prime}+3(2+\xi) \Phi^{\prime}=0,
\end{aligned}
$$

where

$$
\begin{aligned}
& \nu_{1}=2+\xi+\frac{\left[2 m \beta\left(3+2 \epsilon_{\phi}\right) x+\sqrt{6} Q \Omega_{c}\right] x}{\Omega_{c}+2 m \beta x^{2}}, \\
& \nu_{2}=\frac{\Omega_{c} \Psi+2 m \beta x^{2} \delta \varphi_{\mathrm{N}}^{\prime}+\left[2 m \beta\left(3-\xi+2 \epsilon_{\phi}\right) x+\sqrt{6} Q \Omega_{c}\right] x \delta \varphi_{\mathrm{N}}}{\Omega_{c}+2 m \beta x^{2}} .
\end{aligned}
$$

Now, we employ the quasi-static approximation for perturbations deep inside the sound horizon 11, 93. Since the dominant contributions to the perturbation equations are those containing $\mathcal{K}^{2}, \delta_{c \mathrm{~N}}, \delta_{c \mathrm{~N}}^{\prime}$, and $\delta_{b \mathrm{~N}}$, Eqs. $(6.17),(6.22$, and 6.23 give the following relations,

$$
\begin{aligned}
& \Psi=-\Phi \simeq-\frac{3}{2 \mathcal{K}^{2}}\left(\Omega_{c} \delta_{c \mathrm{~N}}+\Omega_{b} \delta_{b \mathrm{~N}}\right) \\
& \delta \varphi_{\mathrm{N}} \simeq \frac{1}{q_{s} \hat{c}_{s}^{2} \mathcal{K}^{2}}\left(m \beta \delta_{c \mathrm{~N}}^{\prime}-\frac{\sqrt{6} Q \Omega_{c}}{2 x} \delta_{c \mathrm{~N}}\right) .
\end{aligned}
$$

Under the quasi-static approximation, we can ignore the terms $3 \Phi^{\prime \prime}, 3 \nu_{1} \Phi^{\prime}$, and $3(2+\xi) \Phi^{\prime}$ in Eqs. 6.30 and (6.31) relative to the others. Substituting Eqs. 6.34, 6.35) and the $N$ derivative of Eq. 6.35) into Eqs. (6.30) and (6.31), it follows that

$$
\begin{aligned}
& \delta_{c \mathrm{~N}}^{\prime \prime}+\nu \delta_{c \mathrm{~N}}^{\prime}-\frac{3}{2 G}\left(G_{c c} \Omega_{c} \delta_{c \mathrm{~N}}+G_{c b} \Omega_{b} \delta_{b \mathrm{~N}}\right) \simeq 0 \\
& \delta_{b \mathrm{~N}}^{\prime \prime}+(2+\xi) \delta_{b \mathrm{~N}}^{\prime}-\frac{3}{2 G}\left(G_{b c} \Omega_{c} \delta_{c \mathrm{~N}}+G_{b b} \Omega_{b} \delta_{b \mathrm{~N}}\right) \simeq 0
\end{aligned}
$$

where

$$
\nu=\frac{2 m \beta(1+2 \beta)\left(5+\xi+2 \epsilon_{\phi}\right) x^{2}+(2+\xi+\sqrt{6} Q x)[1+(2-m) \beta] \Omega_{c}}{2 m \beta(1+2 \beta) x^{2}+\Omega_{c}[1+(2-m) \beta]},
$$

and

$$
\begin{aligned}
G_{c c} & =\frac{1+r_{1}}{1+r_{2}} G, \quad G_{c b}=\frac{1}{1+r_{2}} G, \\
G_{b c} & =G_{b b}=G,
\end{aligned}
$$

with

$$
\begin{aligned}
& r_{1}=\frac{2 Q\left[3 Q \Omega_{c}+\sqrt{6} m \beta x\left(2+\epsilon_{\phi}+\sqrt{6} Q x\right)\right]}{3 \Omega_{c}[1+(2-m) \beta]}, \\
& r_{2}=\frac{2 m \beta(1+2 \beta) x^{2}}{\Omega_{c}[1+(2-m) \beta]} .
\end{aligned}
$$


In the limit that $\beta \rightarrow 0$, we have $r_{1}=2 Q^{2}$ and $r_{2}=0$, so that $G_{c c}=\left(1+2 Q^{2}\right) G$ and $G_{c b}=G_{b c}=G_{b b}=G$. Since $G_{c c}$ is larger than $G$, the growth rate of $\delta_{c \mathrm{~N}}$ is larger than that for $Q=0$. In the other limit $Q \rightarrow 0$, we have $r_{1}=0$ and hence $G_{c c}=G_{c b}=G /\left(1+r_{2}\right)$ and $G_{b c}=G_{b b}=G$. In this case, under the conditions (6.25)-(6.27), both $G_{c c}$ and $G_{c b}$ are smaller than $G$ for $m \beta>0$.

For the theories with $\beta \neq 0$ and $Q \neq 0, G_{c c}$ can be either smaller or larger than $G$ depending on the model parameters. We note that $G_{c b}$ is equivalent to $G_{c c}$ in the limit $Q \rightarrow 0$.

During the $\phi \mathrm{MDE}, G_{c c}$ and $G_{c b}$ are given, respectively, by

$$
\begin{aligned}
G_{c c} & =\left(1+\frac{2 Q^{2}}{1+2 \beta}\right) G \\
G_{c b} & =\left[1-\frac{4 m \beta Q^{2}}{3-2 Q^{2}+6(2-m) \beta^{2}+\left\{12-3 m+(6 m-4) Q^{2}\right\} \beta}\right] G .
\end{aligned}
$$

Under the condition (6.25), $G_{c c}$ is larger than $G$.

A simple approximation valid at the present time for the small couplings $|\beta| \ll 1$ and $|Q| \ll 1$ is given by

$$
G_{c c} \simeq\left(1+2 Q^{2}-\frac{2 \beta m x^{2}}{\Omega_{c}}\right) G
$$

where we ignored the terms which are the products of $\beta$ and $Q$. This shows how $G_{c c}$ can be larger or smaller than $G$ depending on the coupling parameters.

On the fixed point (b) given by Eq. (5.17), $G_{c c}$ reduces to

$$
G_{c c}=\frac{4(1+2 \beta)+\lambda(2 Q-\lambda)}{1+2 \beta} \frac{Q}{\lambda} G
$$

whereas $G_{c b}$ vanishes. From Eq. 6.46 , we have $G_{c c} \rightarrow 0$ for $Q \rightarrow 0$. If we impose the condition $G_{c c}>0$ on the future attractor point $(\mathrm{b})$, we require that $[4(1+2 \beta)+\lambda(2 Q-\lambda)] Q / \lambda>0$.

Since both $G_{c c}$ and $G_{c b}$ are different from $G$, this affects the evolution of $\delta_{c \mathrm{~N}}$ through Eq. 6.36). While both $G_{b c}$ and $G_{b b}$ are equivalent to $G$, the modified evolution of $\delta_{c \mathrm{~N}}$ affects the growth of $\delta_{b \mathrm{~N}}$ through Eq. 6.37). To study the evolution of total matter perturbations, we introduce the effective CDM background density $\hat{\rho}_{c}=\left(1+f_{1}\right) \rho_{c}=$ $e^{Q \phi / M_{\mathrm{pl}}} \rho_{c}$ and the perturbed gauge-invariant density $\hat{\delta \rho_{c \mathrm{~N}}}=e^{Q \phi / M_{\mathrm{pl}}}\left(\delta \rho_{c \mathrm{~N}}+Q \rho_{c} \delta \phi_{\mathrm{N}} / M_{\mathrm{pl}}\right)$. The total matter density contrast is given by

$$
\delta_{m}=\frac{\hat{\delta \rho_{c \mathrm{~N}}}+\delta \rho_{b \mathrm{~N}}}{\hat{\rho}_{c}+\rho_{b}}=\left(\delta_{c \mathrm{~N}}+\sqrt{6} Q x \delta \varphi_{\mathrm{N}}\right) \frac{\Omega_{c}}{\Omega_{m}}+\delta_{b \mathrm{~N}} \frac{\Omega_{b}}{\Omega_{m}}
$$

where $\Omega_{m}=\Omega_{c}+\Omega_{b}$. For the perturbations deep inside the Hubble radius $(\mathcal{K} \gg 1)$, Eq. (6.35) shows that the term $\sqrt{6} Q x \delta \varphi_{\mathrm{N}}$ in Eq. 6.47) is negligibly small relative to $\delta_{c \mathrm{~N}}$, so Eq. 6.47) reduces to $\delta_{m} \simeq \delta_{c \mathrm{~N}} \Omega_{c} / \Omega_{m}+\delta_{b \mathrm{~N}} \Omega_{b} / \Omega_{m}$. The quantity related to the measurement of redshift-space distortions is $f(z) \sigma_{8}(z)$, where $f=\delta_{m}^{\prime} / \delta_{m}$ is the growth rate of matter perturbations that depends on the redshift $z$. On the critical points, $f$ is constant and can be obtained analytically, since all the coefficients of Eqs. 6.36 and (6.37) are constant. If we neglect the contribution of baryons to Eq. 6.30, the value of $f$ corresponding to the growing-mode solution is given by

$$
f=\frac{1}{2}\left(\sqrt{\nu^{2}+6 \frac{G_{c c}}{G} \Omega_{c}}-\nu\right) .
$$

For instance, $f=1+2 Q^{2} / q_{s}$ during the $\phi \mathrm{MDE}$. The matter growth rate is always larger than in standard general relativity $(f=1)$, but, for $\beta>0$, it is smaller than in a pure energy-exchange model with $\beta=0$ and $Q \neq 0$. In general, however, we need the numerical integration to know the precise evolution of $\delta_{m}$ at low redshifts, which we describe next. 

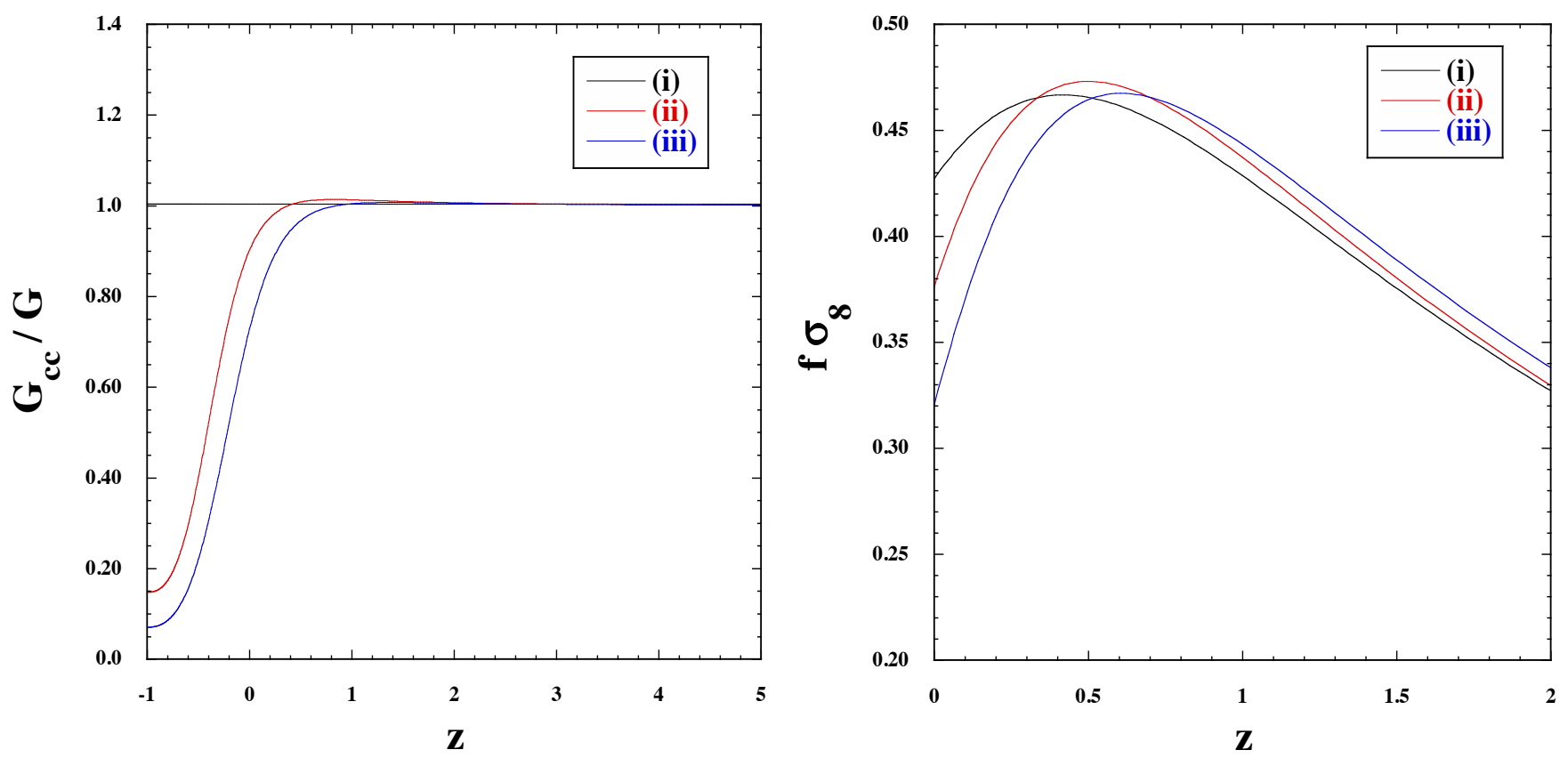

FIG. 2. (Left) Evolution of $G_{c c} / G$ versus the redshift $z$ for $\lambda=1$ in three different cases: (i) $Q=0.04, \beta=0, m=2$, (ii) $Q=0.04, \beta=1, m=2$, and (iii) $Q=0.02, \beta=0.5, m=3$. The background initial conditions are chosen to realize $\Omega_{\phi} \simeq 0.68$, $\Omega_{b} \simeq 0.05$, and $\Omega_{r} \simeq 10^{-4}$ today. (Right) Evolution of $f \sigma_{8}$ versus the redshift $z$ for the three cases shown in the left panel. Today's values of $\sigma_{8}$ and $\mathcal{K}$ are chosen to be $\sigma_{8}(z=0)=0.811$ and $\mathcal{K}(z=0)=300$, respectively.

In Fig. 2, we plot the evolution of $G_{c c} / G$ (left) and $f \sigma_{8}$ (right) versus the redshift $z$ for $\lambda=1$ in three different cases: (i) $Q=0.04, \beta=0, m=2$, (ii) $Q=0.04, \beta=1, m=2$, and (iii) $Q=0.02, \beta=0.5, m=3$. In these cases, the background cosmology corresponds to the $\phi \mathrm{MDE}$ followed by the accelerated fixed point (b). In case (i), all the terms associated with the coupling $\beta$ disappear from the background and perturbation equations, so their dynamics is the same as that of standard coupled quintessence with the exponential potential 43,53 . Indeed, $G_{c c}$ is equivalent to $\left(1+2 Q^{2}\right) G=1.0032 G$ in the simulation of Fig. 2, with $G_{c b}=G$.

In case (ii), the analytic estimations (6.43)- $(6.46)$ show that $G_{c c}=1.0011 G$ and $G_{c b}=0.9986 G$ during the $\phi \mathrm{MDE}$ and $G_{c c}=0.1477 G$ on the accelerated point (b). In comparison to case (i), the existence of positive coupling $\beta$ leads to the smaller value of $G_{c c}$ in $\phi \mathrm{MDE}$, which is also confirmed numerically. At low redshifts, there is a transient period during which $G_{c c}$ gets larger than that in case (i). However, $G_{c c}$ starts to be smaller than $G$ around the redshift $z \lesssim 0.37$ and it reaches today's value $0.90 G$. Thus, even for $Q \neq 0$, the presence of positive coupling $\beta$ can realize $G_{c c}$ smaller than $G$ by today. In case (iii), the decrease of $G_{c c}$ at low redshifts is even more significant relative to case (ii). In this case, today's values of CDM gravitational couplings are $G_{c c}=0.72 G$ and $G_{c b}=0.65 G$ with the future asymptotic value $G_{c c}=0.070 G$.

As we observe in the right panel of Fig. 2, the decrease of $G_{c c}$ less than $G$, which occurs in cases (ii) and (iii) at low redshifts, leads to values of $f \sigma_{8}$ smaller than that in case (i). This suppression of growth of $\delta_{m}$ is the consequence of momentum transfer associated with the positive coupling $\beta$. The evolution of $f \sigma_{8}$ depends on the couplings $Q, \beta$ as well as the model parameters $m, \lambda$. For $Q$ close to $0, G_{c c}$ approaches $G_{c b}=G /\left(1+r_{2}\right)$, which is in the range $G_{c b}<G$ for $m \beta>0$. In such cases, it is easy to realize a CDM gravitational interaction smaller than the Newton gravitational constant. The nonvanishing coupling $Q$ works to enhance $G_{c c}$, but the energy transfer is required for the existence of $\phi \mathrm{MDE}$.

We note that our interacting model with the $\phi \mathrm{MDE}$ is different from the early dark energy recently studied in Ref. [94, in that the latter only modifies the background dynamics with the standard growth of matter perturbations. In our model, the weak cosmic growth rate is realized by the momentum transfer, with the modified early background dynamics by the energy transfer. Hence there is the possibility that the observational tensions of both $\sigma_{8}$ and $H_{0}$ are relaxed. However, clearly, we need detailed Markov chain Monte Carlo simulations with the recent observational data to see whether this is really the case or not. 


\section{CONCLUSIONS}

In this paper, we studied the cosmological dynamics of interacting theories of dark energy and dark matter by paying particular attention to the existence of a scaling $\phi \mathrm{MDE}$. Our goal is not only to alleviate the $H_{0}$ tension problem by realizing the $\phi \mathrm{MDE}$ but also to ease the $\sigma_{8}$ tension problem by achieving a weak gravitational interaction on scales relevant to the growth of large-scale structures. It is possible to satisfy these two demands by considering the interacting action 1.1 containing both energy and momentum transfers. The dependence of $Z$ in the Lagrangian $f_{2}(\phi, X, Z)$, where $Z=u^{\mu} \nabla_{\mu} \phi$ quantifies the interaction between the CDM four velocity $u^{\mu}$ and the scalar derivative $\nabla_{\mu} \phi$, plays a crucial role for the realization of weak gravity.

In Sec. III, we derived the interacting Lagrangian allowing for the existence of scaling solutions which obey the relation $\dot{\phi} / H=$ constant. We showed that the corresponding Lagrangian is of the form (3.12), which contains two arbitrary functions $g_{1}$ and $g_{2}$ with respect to $Y_{1}=X e^{\lambda \phi / M_{\mathrm{pl}}}$ and $Y_{2}=Z e^{\lambda \phi /\left(2 M_{\mathrm{pl}}\right)}$. In Sec. V, we identified the scaling fixed point (a) as well as the other point (b) relevant to late-time cosmic acceleration, without fixing concrete forms of $g_{1}$ and $g_{2}$. We also found that, for models given by the functions (4.28) and (4.29), there exists the scaling $\phi \mathrm{MDE}$ satisfying the relation $w_{\text {eff }}=\Omega_{\phi}=2 Q^{2} /\left(3 q_{s}\right)$. Thus, for the existence of $\phi \mathrm{MDE}$, we require a nonvanishing coupling constant $Q$ associated with the energy transfer.

In Sec. V] we studied the background cosmology for a concrete model given by the interacting Lagrangian (5.3). We showed the existence of $\phi \mathrm{MDE}$ preceded by the radiation fixed point $(5.19)$. As long as the two conditions (5.20) and (5.23) are satisfied, the $\phi \mathrm{MDE}$ is followed by the stable fixed point (5.17) with cosmic acceleration. As we observe in Fig. 11 the field equation of state $w_{\phi}$ is close to 1 in the deep matter era and it approaches the asymptotic value $w_{\phi}=-1+\lambda^{2} /\left(3 q_{s}\right)$ after the temporary approach to -1 at low redshifts. This background dynamics is distinguished from the coupled dark energy scenario with the momentum transfer alone [64].

In Sec. VI, we explored the dynamics of cosmological perturbations for the same interacting model studied in Sec. V. We derived the full linear perturbation equations of motion and applied the quasi-static approximation to the modes deep inside the sound horizon. Under this approximation scheme, the effective gravitational couplings for CDM and baryon density contrasts are given by Eqs. (6.39) and (6.40), respectively. We showed that, depending on model parameters, both $G_{c c}$ and $G_{c b}$ can be smaller than $G$ at low redshifts, while satisfying conditions for the absence of ghosts and Laplacian instabilities. The weak gravitational interaction of CDM leads to the suppression of growth rate of total matter density contrast $\delta_{m}$. As we observe in Fig. 2, this property is attributed to the momentum transfer arising from the coupling $\beta(2 X)^{1-m / 2} Z^{m}$.

We thus showed that the coupled dark energy and dark matter scenario with both energy and momentum exchanges offers an interesting possibility for realizing the $\phi \mathrm{MDE}$ as well as the weak gravitational interaction at low redshifts. The next step is to investigate whether the concrete interacting model proposed in this paper can alleviate the problems of $H_{0}$ and $\sigma_{8}$ tensions present in the $\Lambda$ CDM model. Since the Lagrangian (5.3) contains additional model parameters with respect to those in $\Lambda \mathrm{CDM}$, one might expect that our model is hardly better than the $\Lambda$ CDM from the Bayesian statistical point of view. However, it is known that there are dynamical dark energy models that are comparable with $\Lambda \mathrm{CDM}$ from the point of view of Bayesian statistics, even with more than three additional free parameters [34]. The detailed observational constraint on our interacting dark energy model is left for a future publication.

\section{ACKNOWLEDGEMENTS}

We thank Antonio De Felice and Ryotaro Kase for useful discussions. ST is supported by the Grant-in-Aid for Scientific Research Fund of the JSPS No.19K03854 and MEXT KAKENHI Grant-in-Aid for Scientific Research on Innovative Areas "Cosmic Acceleration" (No. 15H05890).

\section{APPENDIX: PHASE SPACE FOR A SIMPLIFIED CASE}

In this Appendix, we express the dynamical Eqs. $4.4-4.5$ in terms of the dimensionless variables $x, y$, and $\Omega_{m}$ for the scaling Lagrangian (3.12) containing the functions (3.7) and (3.11). In doing so, we use the unit $M_{\mathrm{pl}}=1$ and notations $g_{i, j}=\partial g_{i} / \partial Y_{j}$ and $g_{i, j k}=\partial^{2} g_{i} / \partial Y_{j} \partial Y_{k}$, where $i=1,2$. Then, the autonomous equations are given by

$$
\begin{aligned}
x^{\prime} & =\frac{\sqrt{6} g_{1}\left(x^{2} \Gamma_{2}+\sqrt{6} g_{2} x y^{4}+Q y^{4} \Omega_{m}\right)-\sqrt{6} \Omega_{m} \Gamma_{1}}{2 \Omega_{m}\left(\bar{\Gamma}_{1}+y^{2} g_{1,1}\right)-2 g_{1}\left(x^{2} \bar{\Gamma}_{2}+5 x^{2} y^{2} g_{2,1}+2 \sqrt{2} x y^{3} g_{2,2}+g_{2} y^{4}\right)}+\frac{3 x \Theta}{2 g_{1} y^{2}}, \\
y^{\prime} & =\frac{3 \Theta-\sqrt{6} g_{1} \lambda x y^{2}}{2 g_{1} y},
\end{aligned}
$$




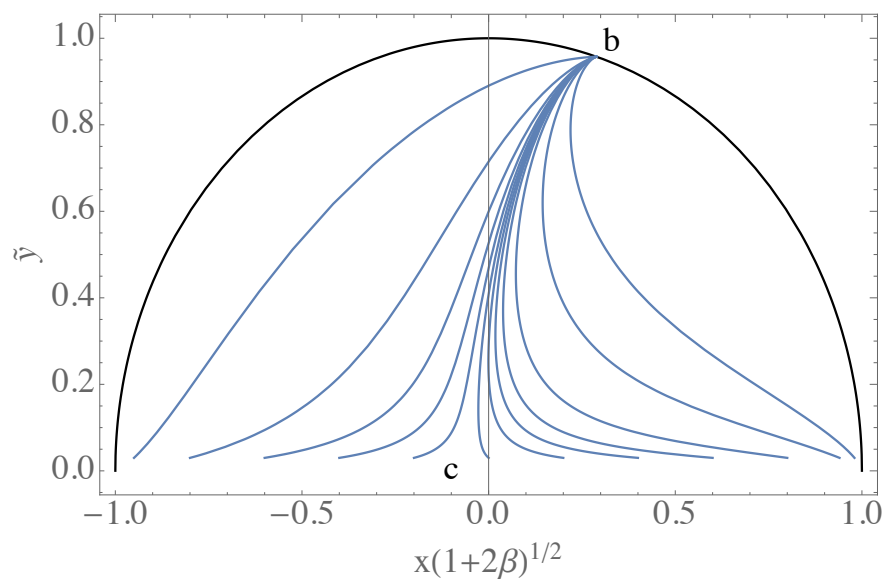

FIG. 3. Phase-space analysis for the same model parameters as those used in Fig. 11 but with neither baryons nor radiation $\left(\Omega_{b}=0\right.$ and $\left.\Omega_{r}=0\right)$. The $\phi \mathrm{MDE}$ saddle point and the final accelerated attractor are denoted as c and b, respectively.

where

$$
\begin{aligned}
\Gamma_{i} & =\lambda x^{2}\left(\bar{\Gamma}_{i}+y^{2} g_{i, 1}\right)+A_{i} y^{2}\left(y g_{i, 2}+\sqrt{2} x g_{i, 1}\right), \\
\bar{\Gamma}_{i} & =2 x^{2} g_{i, 11}+2 \sqrt{2} x y g_{i, 12}+y^{2} g_{i, 22}, \\
A_{1} & =\sqrt{2} Q x-\sqrt{3} w_{m}, \\
A_{2} & =\sqrt{2} \lambda x+\sqrt{3}, \\
\Theta & =g_{1}\left[\sqrt{2} x^{3} y g_{2,2}+2 g_{2} x^{2} y^{2}+y^{2}\left(w_{m}+1\right) \Omega_{m}+2 x^{4} g_{2,1}\right]-x \Omega_{m}\left(2 x g_{1,1}+\sqrt{2} y g_{1,2}\right),
\end{aligned}
$$

and

$$
\Omega_{m}=\frac{g_{1}\left(2 x^{4} g_{2,1}+\sqrt{2} x^{3} y g_{2,2}+g_{2} x^{2} y^{2}-y^{2}\right)}{x\left(2 x g_{1,1}+\sqrt{2} y g_{1,2}\right)-g_{1} y^{2}} .
$$

Let us consider the model given by the functions (5.1) and (5.2). For the perfect fluid, we only take into account CDM with $w_{m}=0$. Then, the autonomous Eqs. $(7.1)$ and $(7.2)$ reduce to

$$
\begin{aligned}
& x^{\prime}=\frac{3}{2} x\left(2 q_{s} x^{2}-2+\Omega_{m}\right)+\frac{\sqrt{6}}{2 q_{s}}\left(\lambda \tilde{y}^{2}-Q \Omega_{m}\right), \\
& \tilde{y}^{\prime}=\frac{1}{2} \tilde{y}\left(6 q_{s} x^{2}-\sqrt{6} \lambda x+3 \Omega_{m}\right),
\end{aligned}
$$

where $\tilde{y}$ is defined by Eq. (5.5), and $\Omega_{m}=1-q_{s} x^{2}-\tilde{y}^{2}$. The dynamical system (7.9)-(7.10) contains the scaling solution (a) characterized by fixed point $\left(x_{c}, y_{c}\right)=\left(\sqrt{6} /[2(Q+\lambda)], \sqrt{\left[2 Q(Q+\lambda)+3 q_{s}\right] /\left[2(Q+\lambda)^{2}\right]}\right)$. Besides this, there are also the fixed point (b) and the $\phi \mathrm{MDE}$ (c) whose values of $x_{c}, y_{c}, \Omega_{\phi}, w_{\text {eff }}$ are the same as those given in Eqs. (5.17) and (5.18), respectively. We also have the kinetic points (d1) and (d2), but they are relevant to neither matter-dominated nor accelerated epochs. As long as the two conditions (5.20) and (5.23) are satisfied, the $\phi \mathrm{MDE}$ is followed by point (b) with cosmic acceleration [instead of the scaling solution (a)]. In Fig. 3, we can confirm that, for the model parameters used in Fig. 1. the solutions converge to the attractor point (b).

[1] P. A. Abell et al. [LSST Science and LSST Project Collaborations], arXiv:0912.0201 [astro-ph.IM].

[2] S. Rawlings, arXiv:1105.6333 [astro-ph.CO].

[3] R. Laureijs et al. [EUCLID Collaboration], arXiv:1110.3193 [astro-ph.CO].

[4] L. Amendola et al. [Euclid Theory Working Group], Living Rev. Rel. 16, 6 (2013) arXiv:1206.1225 [astro-ph.CO]].

[5] M. Levi et al. [DESI Collaboration], arXiv:1308.0847 [astro-ph.CO].

[6] A. G. Riess et al., Astrophys. J. 855, 136 (2018) [arXiv:1801.01120 [astro-ph.SR]]. 
[7] G. W. Horndeski, Int. J. Theor. Phys. 10, 363 (1974).

[8] C. Deffayet, X. Gao, D. A. Steer and G. Zahariade, Phys. Rev. D 84, 064039 (2011) arXiv:1103.3260 [hep-th]].

[9] T. Kobayashi, M. Yamaguchi and J. Yokoyama, Prog. Theor. Phys. 126, 511 (2011) arXiv:1105.5723 [hep-th]].

[10] C. Charmousis, E. J. Copeland, A. Padilla and P. M. Saffin, Phys. Rev. Lett. 108, 051101 (2012) arXiv:1106.2000 [hep-th]].

[11] A. De Felice, T. Kobayashi and S. Tsujikawa, Phys. Lett. B 706, 123 (2011) arXiv:1108.4242 [gr-qc]].

[12] A. De Felice and S. Tsujikawa, JCAP 1202, 007 (2012) arXiv:1110.3878 [gr-qc]].

[13] L. Heisenberg, JCAP 1405, 015 (2014) arXiv:1402.7026 [hep-th]].

[14] G. Tasinato, JHEP 1404, 067 (2014) arXiv:1402.6450 [hep-th]].

[15] A. De Felice, L. Heisenberg, R. Kase, S. Mukohyama, S. Tsujikawa and Y. l. Zhang, JCAP 1606, 048 (2016) arXiv:1603.05806 [gr-qc]].

[16] A. De Felice, L. Heisenberg, R. Kase, S. Mukohyama, S. Tsujikawa and Y. 1. Zhang, Phys. Rev. D 94, 044024 (2016) arXiv:1605.05066 [gr-qc]].

[17] C. de Rham, G. Gabadadze and A. J. Tolley, Phys. Rev. Lett. 106, 231101 (2011) arXiv:1011.1232 [hep-th]].

[18] S. F. Hassan and R. A. Rosen, JHEP 1202, 126 (2012) arXiv:1109.3515 [hep-th]].

[19] P. Creminelli, G. D'Amico, J. Norena and F. Vernizzi, JCAP 0902, 018 (2009) arXiv:0811.0827 [astro-ph]].

[20] G. Gubitosi, F. Piazza and F. Vernizzi, JCAP 1302, 032 (2013) arXiv:1210.0201 [hep-th]].

[21] J. K. Bloomfield, E. E. Flanagan, M. Park and S. Watson, JCAP 1308, 010 (2013) [arXiv:1211.7054 [astro-ph.CO]].

[22] J. Bloomfield, JCAP 1312, 044 (2013) arXiv:1304.6712 [astro-ph.CO]].

[23] J. Gleyzes, D. Langlois, F. Piazza and F. Vernizzi, JCAP 1308, 025 (2013) arXiv:1304.4840 [hep-th]].

[24] B. P. Abbott et al. [LIGO Scientific and Virgo Collaborations], Phys. Rev. Lett. 119, 161101 (2017) arXiv:1710.05832 $[\mathrm{gr}-\mathrm{qc}]]$.

[25] A. Goldstein et al., Astrophys. J. 848, L14 (2017) arXiv:1710.05446 [astro-ph.HE]].

[26] P. Creminelli and F. Vernizzi, Phys. Rev. Lett. 119, 251302 (2017) arXiv:1710.05877|[astro-ph.CO]].

[27] J. M. Ezquiaga and M. Zumalacarregui, Phys. Rev. Lett. 119, 251304 (2017) arXiv:1710.05901 [astro-ph.CO]].

[28] J. Sakstein and B. Jain, Phys. Rev. Lett. 119, 251303 (2017) arXiv:1710.05893 [astro-ph.CO]].

[29] T. Baker, E. Bellini, P. G. Ferreira, M. Lagos, J. Noller and I. Sawicki, Phys. Rev. Lett. 119, 251301 (2017) arXiv:1710.06394 [astro-ph.CO]].

[30] M. Crisostomi and K. Koyama, Phys. Rev. D 97, 084004 (2018) arXiv:1712.06556 [astro-ph.CO]].

[31] R. Kase and S. Tsujikawa, Phys. Rev. D 97, 103501 (2018) arXiv:1802.02728 [gr-qc]].

[32] L. Amendola, M. Kunz, I. D. Saltas and I. Sawicki, Phys. Rev. Lett. 120, 131101 (2018) arXiv:1711.04825 [astro-ph.CO]].

[33] R. Kase and S. Tsujikawa, Int. J. Mod. Phys. D 28, 1942005 (2019) arXiv:1809.08735 [gr-qc]].

[34] S. Nakamura, A. De Felice, R. Kase and S. Tsujikawa, Phys. Rev. D 99, 063533 (2019) arXiv:1811.07541 [astro-ph.CO]].

[35] P. A. R. Ade et al. [Planck Collaboration], Astron. Astrophys. 594, A13 (2016) arXiv:1502.01589 [astro-ph.CO]].

[36] L. Verde, T. Treu and A. G. Riess, Nature Astronomy, 3, 891-895 (2019) arXiv:1907.10625 [astro-ph.CO]].

[37] A. G. Riess, S. Casertano, W. Yuan, L. M. Macri and D. Scolnic, Astrophys. J. 876, no. 1, 85 (2019) arXiv:1903.07603 [astro-ph.CO]].

[38] W. L. Freedman et al., arXiv:1907.05922 [astro-ph.CO].

[39] M. J. Reid, D. W. Pesce and A. G. Riess, Astrophys. J. 886, no. 2, L27 (2019)

[40] C. Heymans et al., Mon. Not. Roy. Astron. Soc. 427, 146 (2012) arXiv:1210.0032 [astro-ph.CO]].

[41] H. Hildebrandt et al., Mon. Not. Roy. Astron. Soc. 465, 1454 (2017) arXiv:1606.05338 [astro-ph.CO]].

[42] C. Wetterich, Astron. Astrophys. 301, 321 (1995) hep-th/9408025.

[43] L. Amendola, Phys. Rev. D 62, 043511 (2000) astro-ph/9908023.

[44] V. Pettorino, Phys. Rev. D 88, 063519 (2013) arXiv:1305.7457 [astro-ph.CO]].

[45] P. A. R. Ade et al. [Planck Collaboration], Astron. Astrophys. 594, A14 (2016) arXiv:1502.01590 [astro-ph.CO]].

[46] C. Brans and R. H. Dicke, Phys. Rev. 124, 925 (1961).

[47] L. Amendola, Phys. Rev. D 60, 043501 (1999) astro-ph/9904120.

[48] J. Khoury and A. Weltman, Phys. Rev. Lett. 93, 171104 (2004) astro-ph/0309300.

[49] S. Tsujikawa, K. Uddin, S. Mizuno, R. Tavakol and J. Yokoyama, Phys. Rev. D 77, 103009 (2008) arXiv:0803.1106 [astro-ph]].

[50] B. F. Schutz and R. Sorkin, Annals Phys. 107, 1 (1977).

[51] J. D. Brown, Class. Quant. Grav. 10, 1579 (1993) gr-qc/9304026.

[52] N. Frusciante, R. Kase, K. Koyama, S. Tsujikawa and D. Vernieri, Phys. Lett. B 790, 167 (2019) arXiv:1812.05204 [gr-qc]].

[53] L. Amendola, Phys. Rev. D 69, 103524 (2004) astro-ph/0311175.

[54] A. Pourtsidou, C. Skordis and E. J. Copeland, Phys. Rev. D 88, 083505 (2013) arXiv:1307.0458 [astro-ph.CO]].

[55] C. G. Boehmer, N. Tamanini and M. Wright, Phys. Rev. D 91, 123002 (2015) arXiv:1501.06540 [gr-qc]].

[56] C. G. Boehmer, N. Tamanini and M. Wright, Phys. Rev. D 91, 123003 (2015) arXiv:1502.04030 [gr-qc]].

[57] C. Skordis, A. Pourtsidou and E. J. Copeland, Phys. Rev. D 91, 083537 (2015) arXiv:1502.07297 [astro-ph.CO]].

[58] T. S. Koivisto, E. N. Saridakis and N. Tamanini, JCAP 1509, 047 (2015) arXiv:1505.07556 [astro-ph.CO]].

[59] A. Pourtsidou and T. Tram, Phys. Rev. D 94, 043518 (2016) arXiv:1604.04222 [astro-ph.CO]].

[60] J. Dutta, W. Khyllep and N. Tamanini, Phys. Rev. D 95, 023515 (2017) arXiv:1701.00744 [gr-qc]].

[61] M. S. Linton, A. Pourtsidou, R. Crittenden and R. Maartens, JCAP 04, 043 (2018) arXiv:1711.05196 [astro-ph.CO]].

[62] R. Kase and S. Tsujikawa, Phys. Rev. D 101, 063511 (2020) arXiv:1910.02699 [gr-qc]].

[63] F. N. Chamings, A. Avgoustidis, E. J. Copeland, A. M. Green and A. Pourtsidou, arXiv:1912.09858 [astro-ph.CO].

[64] R. Kase and S. Tsujikawa, Phys. Lett. B 804, 135400 (2020) arXiv:1911.02179 [gr-qc]]. 
[65] N. Dalal, K. Abazajian, E. E. Jenkins and A. V. Manohar, Phys. Rev. Lett. 87, 141302 (2001) astro-ph/0105317.

[66] W. Zimdahl and D. Pavon, Phys. Lett. B 521, 133 (2001) astro-ph/0105479.

[67] L. P. Chimento, A. S. Jakubi, D. Pavon and W. Zimdahl, Phys. Rev. D 67, 083513 (2003) astro-ph/0303145.

[68] B. Wang, Y. g. Gong and E. Abdalla, Phys. Lett. B 624, 141 (2005) hep-th/0506069.

[69] H. Wei and S. N. Zhang, Phys. Lett. B 644, 7 (2007) astro-ph/0609597.

[70] L. Amendola, G. Camargo Campos and R. Rosenfeld, Phys. Rev. D 75, 083506 (2007) astro-ph/0610806.

[71] Z. K. Guo, N. Ohta and S. Tsujikawa, Phys. Rev. D 76, 023508 (2007) astro-ph/0702015 [astro-ph]].

[72] J. Valiviita, E. Majerotto and R. Maartens, JCAP 0807, 020 (2008) arXiv:0804.0232 [astro-ph]].

[73] M. B. Gavela, D. Hernandez, L. Lopez Honorez, O. Mena and S. Rigolin, JCAP 0907, 034 (2009) arXiv:0901.1611 [astro-ph.CO]].

[74] V. Salvatelli, N. Said, M. Bruni, A. Melchiorri and D. Wands, Phys. Rev. Lett. 113, 181301 (2014) arXiv:1406.7297 [astro-ph.CO]].

[75] S. Kumar and R. C. Nunes, Phys. Rev. D 94, 123511 (2016) arXiv:1608.02454 [astro-ph.CO]].

[76] E. Di Valentino, A. Melchiorri and O. Mena, Phys. Rev. D 96, 043503 (2017) arXiv:1704.08342 [astro-ph.CO]].

[77] R. An, C. Feng and B. Wang, JCAP 1802, 038 (2018) arXiv:1711.06799 [astro-ph.CO]].

[78] W. Yang, S. Pan, E. Di Valentino, R. C. Nunes, S. Vagnozzi and D. F. Mota, JCAP 1809, 019 (2018) arXiv:1805.08252 [astro-ph.CO]].

[79] S. Pan, W. Yang, E. Di Valentino, E. N. Saridakis and S. Chakraborty, arXiv:1907.07540 [astro-ph.CO].

[80] E. Di Valentino, A. Melchiorri, O. Mena and S. Vagnozzi, arXiv:1908.04281 [astro-ph.CO].

[81] W. Yang, S. Pan, R. C. Nunes and D. F. Mota, arXiv:1910.08821 [astro-ph.CO].

[82] F. Piazza and S. Tsujikawa, JCAP 0407, 004 (2004) hep-th/0405054.

[83] S. Tsujikawa and M. Sami, Phys. Lett. B 603, 113 (2004) hep-th/0409212.

[84] L. Amendola, M. Quartin, S. Tsujikawa and I. Waga, Phys. Rev. D 74, 023525 (2006) astro-ph/0605488.

[85] S. Tsujikawa, Phys. Rev. D 73, 103504 (2006) hep-th/0601178.

[86] E. J. Copeland, A. R. Liddle and D. Wands, Phys. Rev. D 57, 4686 (1998) gr-qc/9711068.

[87] T. Barreiro, E. J. Copeland and N. J. Nunes, Phys. Rev. D 61, 127301 (2000) astro-ph/9910214].

[88] E. J. Copeland, M. Sami and S. Tsujikawa, Int. J. Mod. Phys. D 15, 1753 (2006) hep-th/0603057].

[89] L. Amendola, D. Bettoni, G. Domnech and A. R. Gomes, JCAP 1806, 029 (2018) arXiv:1803.06368 [gr-qc]].

[90] N. Frusciante, R. Kase, N. J. Nunes and S. Tsujikawa, Phys. Rev. D 98, 123517 (2018) arXiv:1810.07957][gr-qc]].

[91] S. Savastano, L. Amendola, J. Rubio and C. Wetterich, Phys. Rev. D 100, 083518 (2019) arXiv:1906.05300 [astro-ph.CO]].

[92] R. Kase and S. Tsujikawa, arXiv:2005.13809 [gr-qc].

[93] B. Boisseau, G. Esposito-Farese, D. Polarski and A. A. Starobinsky, Phys. Rev. Lett. 85, 2236 (2000) gr-qc/0001066.

[94] J. C. Hill, E. McDonough, M. W. Toomey and S. Alexander, arXiv:2003.07355 [astro-ph.CO]. 